\title{
1 The Effects of Institutions on Migrant Wages in China and Indonesia
}

Paul Frijters, Xin Meng and Budy Resosudarmo

\section{Introduction}

According to Bell and Muhidin (2009) of the UN Development Programme (UNDP), '[i]nternal migration is the most significant process driving changes in the pattern of human settlement across much of the world, yet remarkably few attempts have been made to compare internal migration between countries'. They estimate that nearly 800 million individuals are internal migrants who live in a different region than the one in which they were born, compared with 'merely' 200 million migrants who have moved countries. The World Bank Development Report 2009 calls this internal migration one of the key drivers of world economic prosperity.

One important aspect of migration is that of the labour-market outcomes of migrants versus their urban incumbents. The literature on migration has always argued that migrants are a selected group. On average, migrants are more motivated, and hence, other things being equal, should do better than locals in the receiving labour market. Most studies comparing labour-market outcomes for migrants and natives often find, however, that migrant outcomes are worse than those of their native counterparts. The explanations for this puzzle include the lack of local labour-market knowledge and communication skills on the migrants' part (see, for example, Borjas 2003; Card 2009; Card et al. 2009; Ottaviano and Peri 2006) and discrimination on the employers' part (Becker 1971).

Most studies have analysed outcomes for people moving from one country to another-a situation under which the lack of local language, local information and discrimination should matter a great deal and might be expected to more than offset the impact of selection. The positive effect of selection should be more evident when analysing internal migration, as migrants and locals share a common language and culture, though levels of education and experience are still likely to differ. Similarly, we might expect discrimination to play a lesser role unless the internal migrants come from a particular (different) ethnic group or when there are particular institutional restrictions that disadvantage migrants.

In this chapter, we study large internal migrant movements in China and Indonesia, using a unique data set on migrants with consistent questionnaires for both countries. In each country, we compare labour-market outcomes of rural-to-urban migrants with those of their city incumbents. In China, rural-urban migrants face very strong legal labour-market discrimination: they are restricted by the type of jobs they may obtain and do not have access to the social welfare available to urban incumbents. In Indonesia, there is almost no legal distinction between migrants and incumbents. 
The key statistical difficulty in comparing labour-market outcomes lies in overcoming the issue of migrant selectivity. We lack clear natural experiments in our data, but we are able to map the migration history of the migrants' region of origin into our data sets. This allows us to use the proportion of previous migrants from a particular region to the cities as an instrument. It is often found in developing countries that the lack of formal information channels makes migration flows heavily dependent upon informal information or, in other words, personal contacts (see, for example, Banerjee 1984; Meng 2000). We believe that the lagged information flow will have a trended impact on the probability of migration mainly via non-wage factors, such as whether migrants can find a home, whether their children feel comfortable and whether someone can help them find a job. Such advantages of prior migration from one's own region make migration more likely without a direct effect on wages.

Although in both countries migrants earn more in the cities than the average rural worker, we find that migrants in China receive significantly lower hourly earnings than their urban incumbents (50 per cent) while migrants in Indonesia receive significantly higher earnings (30 per cent). Selection in both countries is strongly positive while occupational selectivity is negative in China (where migrants are not allowed to work in some of the more high-earning sectors) and positive in Indonesia. As a result, the unexplained portion of differences in hourly wages between migrants and city incumbents is close to zero in Indonesia while it is close to 50 per cent in China. We attribute the latter to the discriminatory legislation in place in most Chinese cities during the survey period.

\section{Background}

During the past 20 years or so, the unprecedented economic growth in China and Indonesia has been accompanied by a large population movement from the countryside to the cities. Between 1995 and 2007, the number of rural-to-urban migrants in China increased from 40 million to about 150 million, accounting for 20 per cent of the rural labour force and one-third of the total urban labour force. Since independence in 1949, the official urban population in Indonesia grew by about $4-5$ per cent per annum - two to three times the growth in total population. In 2005 migrants accounted for about 20 per cent of the total urban population in Indonesia (Meng and Manning 2009).

One explanation for this large-scale internal migration is the large rural-urban income gap; urban Chinese per capita income and per capita expenditure were 2.6 and 2.1 times that of their rural counterparts in 2007. In Indonesia, the ratio for household expenditure was 1.8 in 2008. Qualitatively, these differences in both countries seem large enough to entice the more talented rural workers to move to cities for financial gain, which is indeed borne out by the data that show that the young and more educated rural workers go to the cities (see below).

While both China and Indonesia face similar challenges in terms of transitioning away from a predominantly agricultural economy to one dominated by city-based services and industry, the institutions surrounding internal migration are very different. China, for its part, has established tight controls on the migration process, preventing overly rapid migration as well as forcing migrants to keep ties with their home villages. The most 
important restriction has been that migrants moving to cities are, in general, not allowed to have a city 'citizenship' (hukou). Such intra-country 'citizenship' matters for work and everyday life: migrants are restricted in the type of jobs they may take and, in most cities, are only permitted to take jobs that urban incumbents are unwilling to take. Even if they do the same job as someone with urban citizenship, migrants are not entitled to employer contributions to health insurance, unemployment insurance, housing subsidies or pension coverage. The disadvantages of lacking urban citizenship also extend to the families of migrants: children of migrant workers are not allowed to enroll in normal city schools without paying extra fees (Du et al. 2006; Meng 2000; Meng and Manning 2009; Meng and Zhang 2001; West and Zhao 2000). As a consequence, rural-urban migration in China follows a 'guest worker' system where children remain in the countryside and migrants remain only temporarily in the cities.

Rural-urban migration in Indonesia follows quite different patterns. In contrast with China, in Indonesia, the various governments in the past 40 years have placed very few restrictions on rural-urban migration, with rural and urban citizens treated more or less equally. The only important restriction on migration is that citizens are not permitted to have dual residential cards (kartu tanda penduduk or KTP) - that is, one may not be a resident in two different places. Residency status, however, has no significant labourmarket consequences in Indonesia. ${ }^{1}$ Not having a city residential card does not restrict one from obtaining particular jobs in the city, nor does it restrict one's access to public facilities such as health centres and schools. Even the restrictions on residential ownership are easy to bypass. It is not costly to bribe the local authority at the place of origin to provide a fake letter stating that one has relinquished one's rural residential status or to bribe the local authority in the city to obtain a city residential card without showing proof of the release of one's rural residential status. Thus even the few restrictions that exist turn out not to be binding.

To sum up, both China and Indonesia have had large-scale rural-to-urban migration in the past 20 or so years, but migrants face very different institutional settings in the two countries. As a result of the laissez-faire institutions in Indonesia compared with China, we expect that the labour-market outcomes of migrants vis-a-vis urban incumbents are better in Indonesia.

\section{A statistical model of migration}

Individual $i$ 's hourly log-wages, $\ln \left(y_{i}\right)$, are assumed to be generated by an endowment of observable productivity characteristics, $x_{i}$ (such as education), migration status and an unobserved productivity characteristic, $u_{i}$ (which does not depend on $x_{i}$ or on initial location) (Equation 15.1).

Equation 15.1

$$
\ln \left(y_{i}\right)=x \beta+\text { Migrant } * f+v_{i}
$$


In Equation 15.1, Migrant ${ }_{i}$ is a dummy variable equal to 1 if the person is a migrant and equal to 0 if the person in the wage regression was born in the city. Hence, in this regression, the sample base is the city. Here, $\beta$ denotes the return to observable characteristics in the city. The parameter $f<0$ denotes the degree of discrimination (exploitation) arising from actual restrictions on migrants in the cities. Ex ante, we expect $f$ to be close to zero in a laissez-faire country such as Indonesia and to be significantly negative in a country with legal discrimination such as China. We assume that the decision to migrate is generated by a latent variable process (Equation 15.2).

Equation 15.2

Migrantrural $_{i}-1 \Leftrightarrow x_{i} \delta_{0}+z \delta_{1}+e_{i}>0, \quad e_{i} \sim N(0,1)$

In Equation 15.2, $z_{i}$ contains an appropriate instrument related to the decision to migrate but not directly effecting wages. In this regression, Migrantrural $_{i}$ is a dummy variable equal to 1 if the person is a migrant living in the city and it is 0 if the person still lives in the countryside. The selectivity problem in Equation 15.1 stems from the likelihood that $v_{i}$ and $e_{i}$ are positively related in that the more able potential migrants will actually migrate.

Given estimates for and $\delta_{0}$ and $\delta_{1}$, we can then overcome the selection issue in a wage equation by running the regression (Equation 15.3).

Equation 15.3

$\ln \left(y_{j}\right)=x \beta+$ Migrant $_{i} * f+$ Migrant $_{j} * \hat{\lambda}_{i}+v_{\text {, }}$

The variable is the inverse-Mills ratio of the selection equation and is equal to $\phi\left(x \hat{\delta}_{0}+z \hat{\delta}_{1}\right)$. The identifying assumption that yields the effect of migration is that the $\Phi\left(x \hat{\delta}_{0}+z \hat{\delta}_{1}\right)$ innate characteristics, $e_{i^{\prime}}$ have the same distribution at birth in any region individuals who were born in the city. ${ }^{2}$

\section{Data}

The data used in this study are drawn mainly from the Rural-Urban Migration in China and Indonesia (RUMiCI) survey, which was conducted between March and July 2008. Detailed information on the sampling process of the survey in both countries can be found in Meng and Manning (2009).

The China survey comprises three independent samples: a Rural Household Survey (RHS) with 8000 households, an Urban Household Survey (UHS) with 5000 households, and an Urban Migrant Survey (UMS) with 5000 households. The RHS was conducted in 11 provinces while the UHS and UMS were conducted in 15 cities of nine different provinces. ${ }^{3}$ While households from the rural sample cannot be linked to households in the migrant sample, both the rural and the migrant surveys contained similar information regarding individual and village characteristics. What this means is that the selection in Equation 15.2 uses a different sample of migrants to the eventual wage Equation 15.3; the selection equation will use information on migrants collected in the rural villages, where the questionnaire was 
filled in either by the migrants (when they were visiting home) or by their family members. In order to ascertain whether this brings in any bias, we will compare the characteristics of these proxy migrants with the characteristics of the migrants interviewed in the cities (the UMS). Nevertheless, since more than 70 per cent of the migrants surveyed in the 15 cities came from the nine provinces where we conducted the rural survey, we can use the information collected in the RHS to obtain predicted migration probabilities for more than 70 per cent of the migrants in the UMS sample.

The RUMiCI survey in Indonesia was conducted only in urban areas. Approximately 2500 households in total from four cities (Medan, Tangerang, Samarinda and Makassar) were surveyed, including 921 urban households, 922 lifetime migrant households, and 594 recent migrant households. A migrant is defined as someone who spent at least five years in a rural area before completing primary school. Those who moved to cities in the past five years are defined as recent migrants and the rest are termed lifetime migrants. This distinction is important in Indonesia since one can expect the characteristics of the earlier migrants (in terms of house ownership and job status) to be closer to their urban counterparts. It is the recent migration that is comparable with migration in China and that we will mainly focus on. The sampling frame started with a mini-census conducted in early 2008 consisting of all households in the listing of the 2007 National Socio-Economic Survey (Survei Sosial Ekonomi Nasional, or Susenas) for the cities of Medan, Tangerang, Samarinda and Makassar. This mini-census provides information on the size of each type of migrant household in all cities as well as their regions of origin. We then mapped household information in rural areas corresponding with the rural origins of migrant households in the RUMiCI survey from the 2007 National Socio-Economic Survey, which was taken in July-August 2007. The mapped rural sample consists of 28805 households. Hence, in the Indonesian case we directly compare the migrants in the city with the remaining rural population in the region of origin.

The summary statistics for the matched rural samples for China and Indonesia are reported in Tables $15.1 \mathrm{a}$ and $15.1 \mathrm{~b}$, respectively. Table 15.1a shows that for the China sample, 28 per cent were migrants in 2007. Relative to those who did not migrate, migrants are about 10 years younger, 10 per cent more likely to be male and have about one additional year of schooling. Although migrants are more educated, on average, their self-assessed school performance does not differ much. We also find that migrants are healthier, about $1 \mathrm{~cm}$ taller than non-migrants, are less likely to be married and, on average, have fewer children. We also present some village-level information and find that migrants are more likely to come from villages where the daily wage for unskilled labour is lower and where more people migrated three years ago. The daily wage for unskilled labour will be used as a control in the selection equation, while the percentage of prior migration is our main instrument.

The sample statistics for Indonesia are in Table 15.1b. In the unweighted combined sample, only 3 per cent are migrants, but these migrants are younger with almost two more years of education on average than their rural counterparts (although this is somewhat offset by the fact that 30 per cent of the rural sample are 'students' and therefore still in school). 
Rising China: Global Challenges and Opportunities

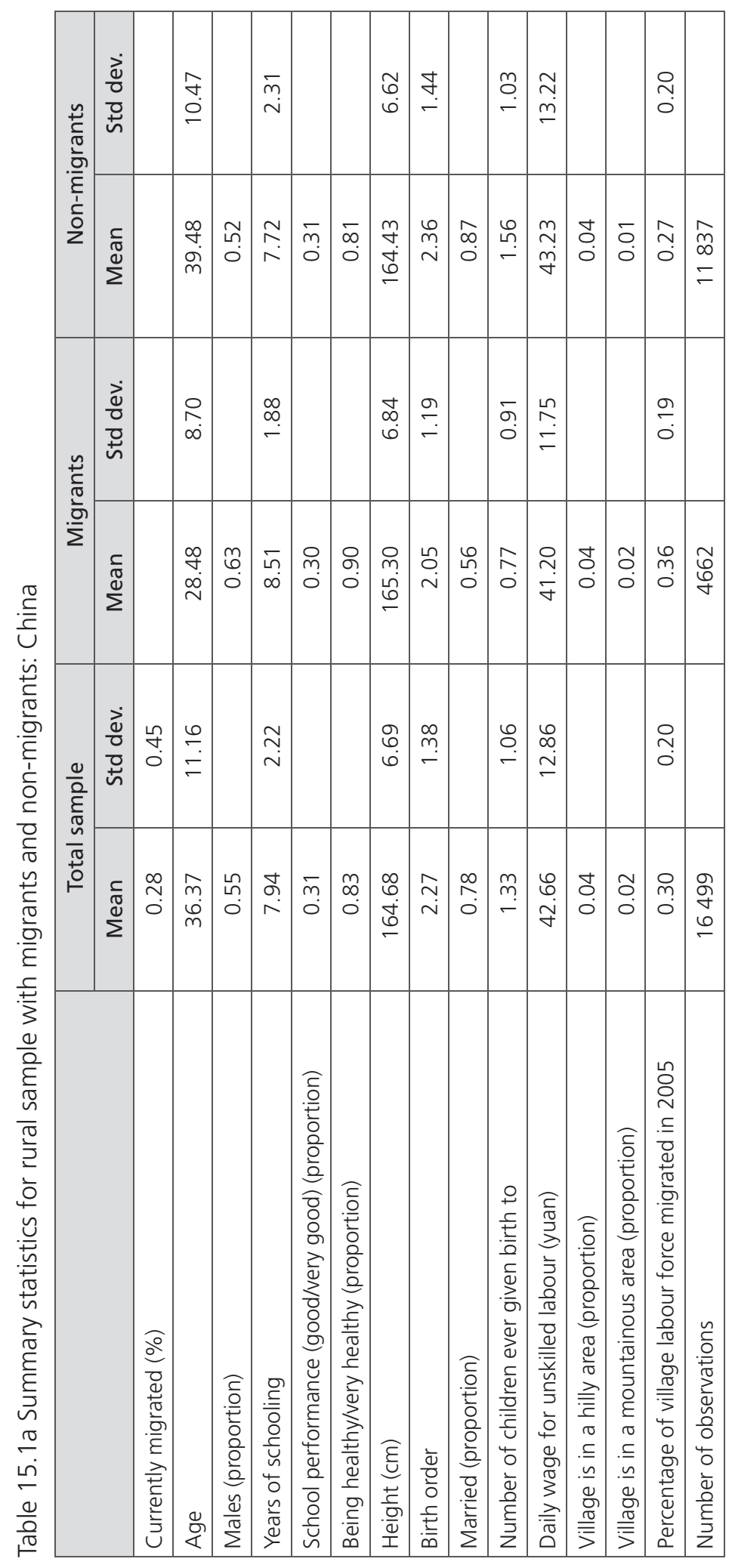




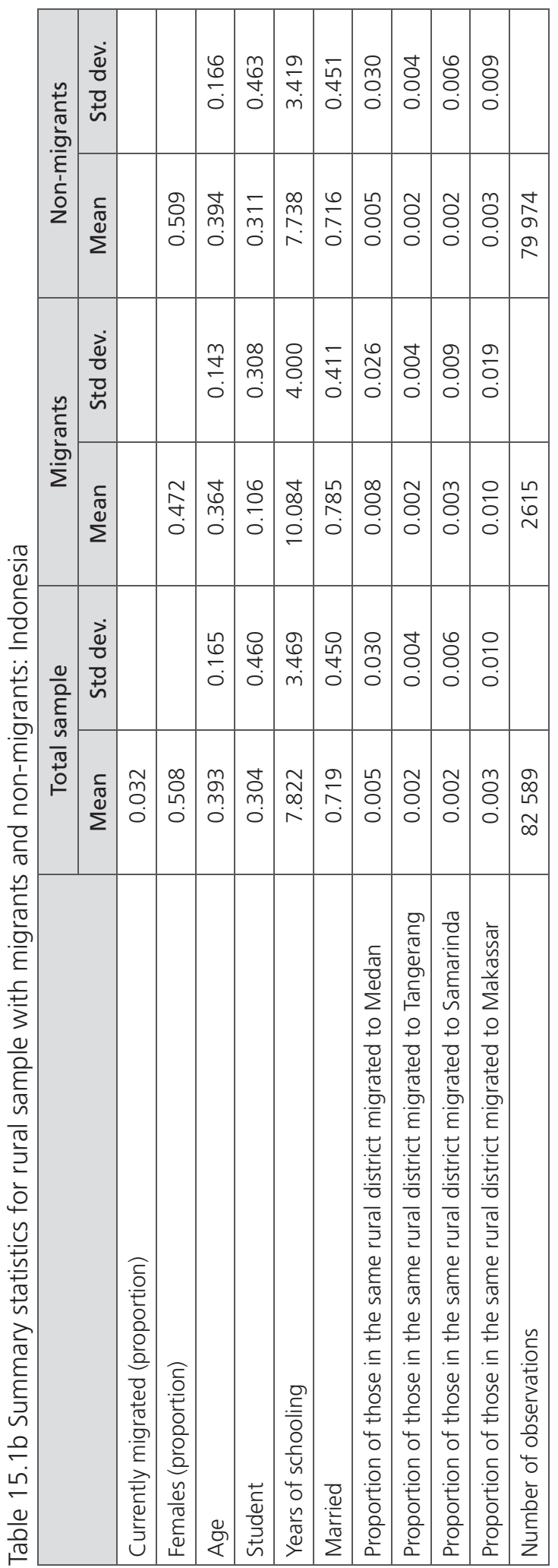


Before we launch into a comparison of migrants and urban incumbents, we present some graphs to show that the migrants observed in the RHS are similar in characteristics to the migrants observed in the UMS. Figure 15.1 plots the age, years of schooling, and height distributions for the two migrant samples for China and it shows that migrants in both samples have similar age and height distributions, but migrants observed in cities are more educated than those observed in the rural areas. On average, the difference in years of schooling is about 0.9 of a year. Since we have only one survey of migrants in Indonesia (there is no implied set of migrants from the countryside, only the migrants found in the cities) there is no concomitant figure for Indonesia.

Figure 15.1 Comparison between migrants in rural and urban surveys: China

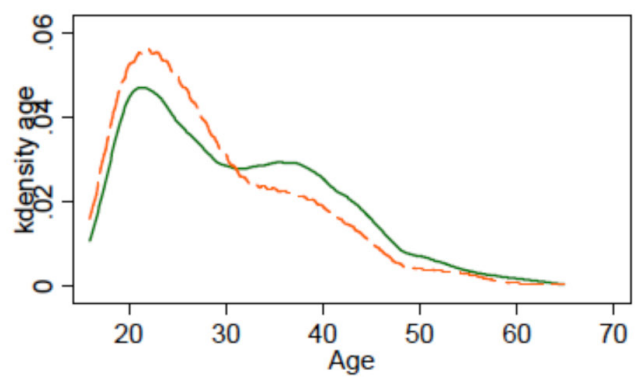

urban migrant - rural migrant

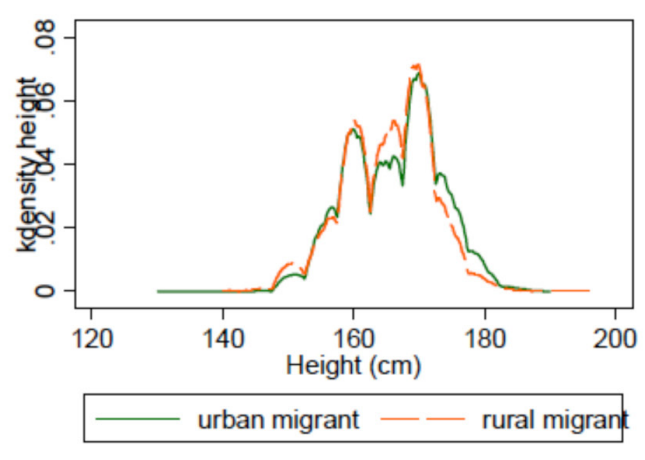

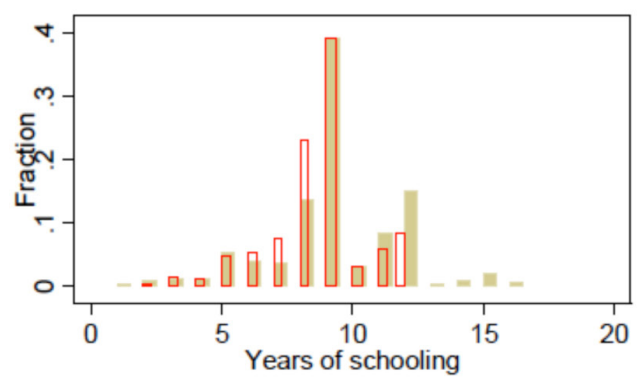

\begin{tabular}{|l|l}
\hline urban migrant \\
\end{tabular}

Tables $15.2 \mathrm{a}$ and $15.2 \mathrm{~b}$ summarise the unconditional means of important variables for migrants and locals in the cities for the two countries. The China sample shown in Table 15.2a includes individuals who are in the labour force between the ages of sixteen and fifty-five and have positive earnings. On average, migrants in our sample are 11 years younger than their urban incumbents, 5 per cent more likely to be males, with one year less schooling, 10 per cent more likely to be healthy, and almost equal in height. With regard to labour-market outcomes, migrants on average earn about 65 per cent of urban incumbents' earnings, and the two groups have very different occupational distributions. Figure 15.2a exhibits the occupational distribution for the migrant and urban samples in China, whilst Figure 15.2b shows the distribution for Indonesia. Figure 15.2a clearly shows that most migrants in China are concentrated in the sales-services and production workers categories, while most of the urban incumbents are in the professional, managerial and clerical groups. 
In Indonesia, the occupational groupings of migrants and urban incumbents look far more similar, with services and skilled workers dominating the distribution. The percentage of professionals is exactly the same in Indonesia for migrants and non-migrants.

Figure 15.2a Occupational distribution of migrants and urban incumbents: China

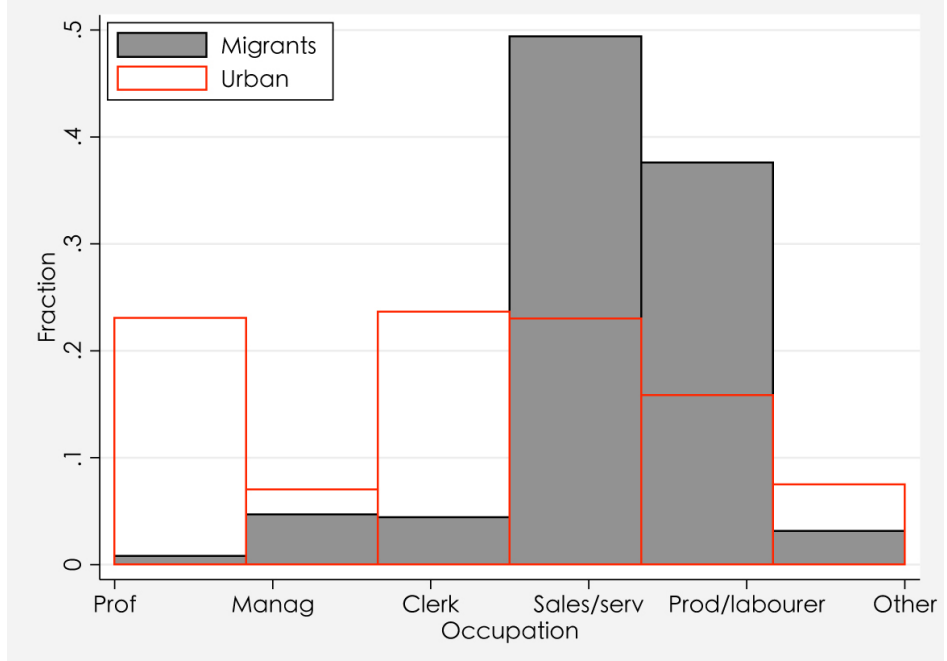

Figure 15.2b Occupational distribution of migrants and urban incumbents: Indonesia

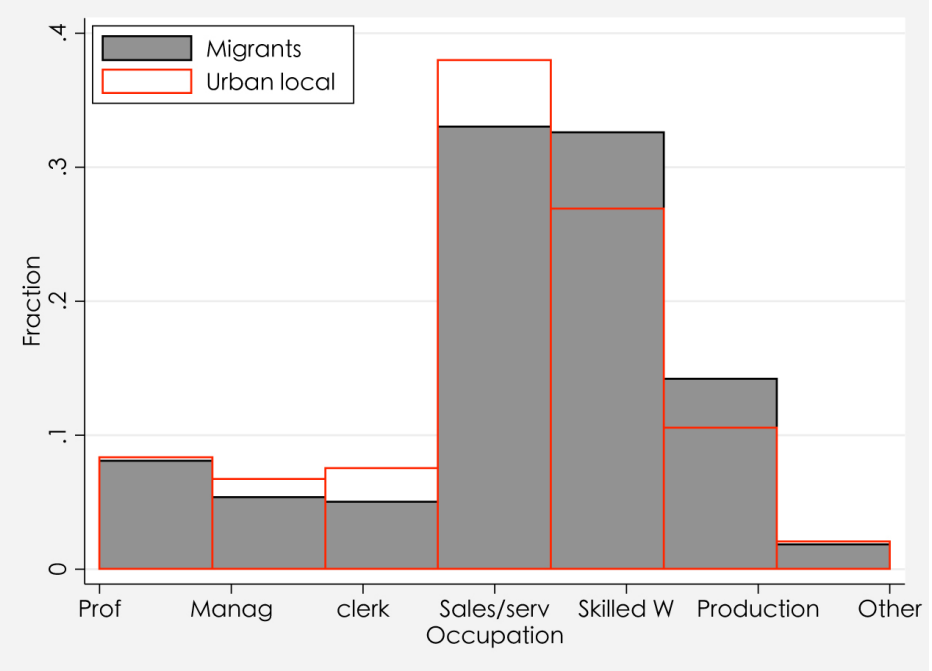

Table $15.2 \mathrm{~b}$ shows the averages for migrants and urban incumbents in Indonesian cities. The Indonesian data cover individuals who are in the labour force between the ages of sixteen and eighty-six with positive earnings. In general, there is no clear difference between the characteristics of migrants and those of locals in the cities, with the only significant differences being that migrants in our sample are 8 per cent more likely to be males than their urban incumbents and 5 per cent more likely to be smokers. Differences in education or health are otherwise small. 


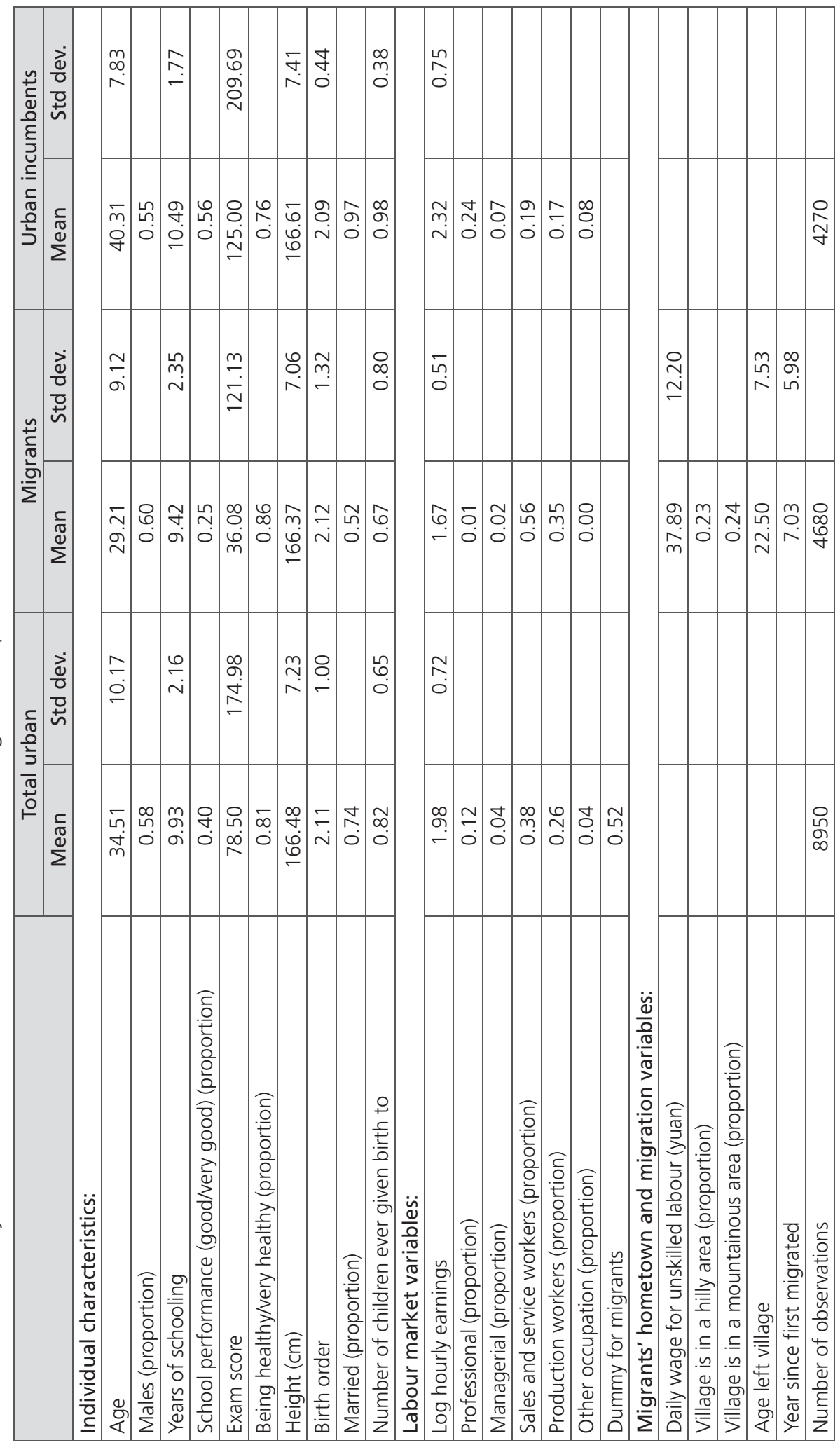




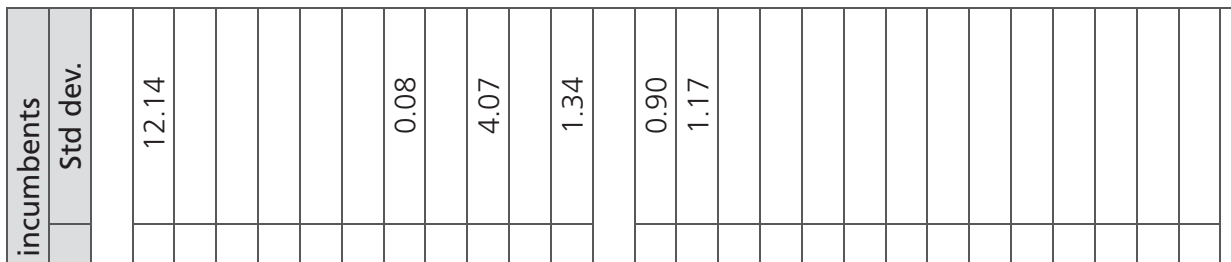

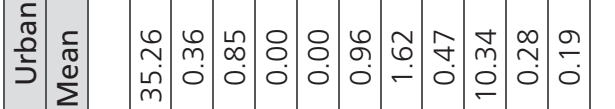

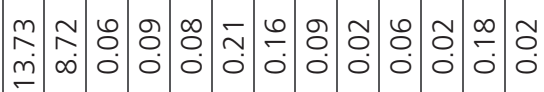
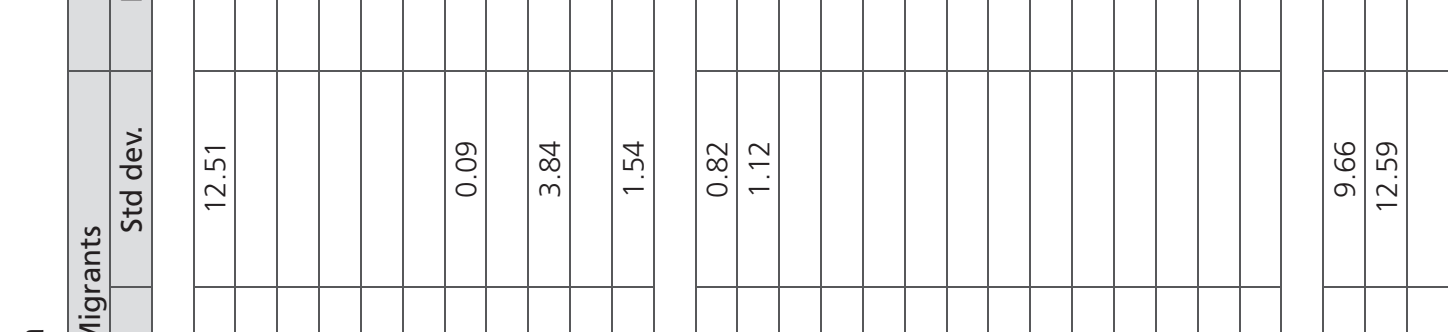

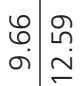

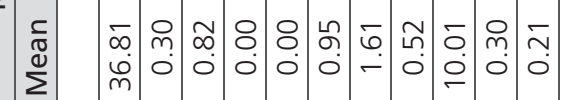

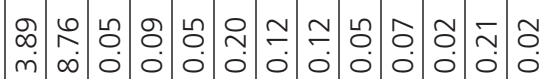

$\underset{m}{m} \stackrel{m}{\sim} \infty$

ㅇํำ

i்

을

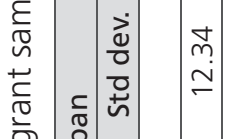

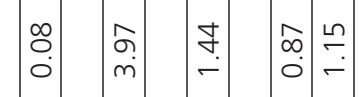

$\infty$ :

$\stackrel{\sim}{\sim}$

흘

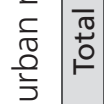

䍃

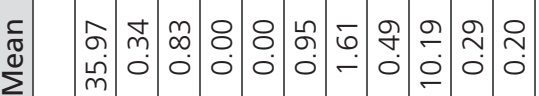

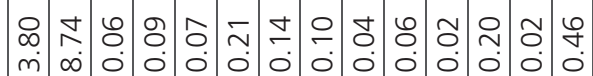

ㅇํ ต

음

先

है

ह

$\subseteq$

$\frac{1}{\pi}$
$\frac{1}{2}$
$\frac{1}{5}$

흐

y

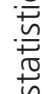

$\frac{2}{0}$

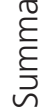

으

ㄷำ

$\frac{1}{\frac{0}{0}}$

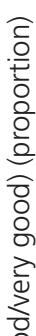

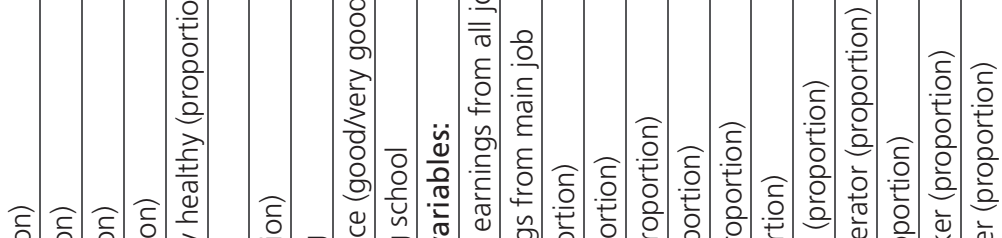

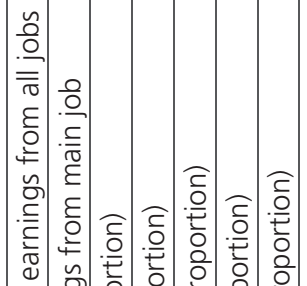

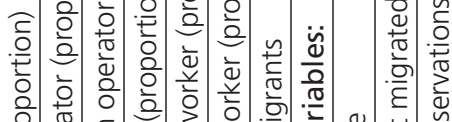

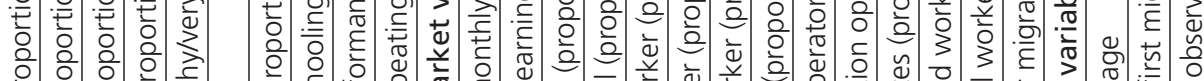

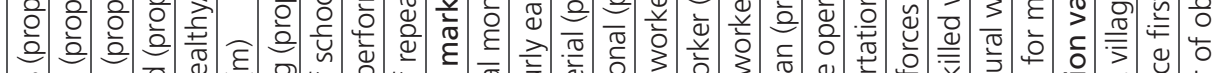

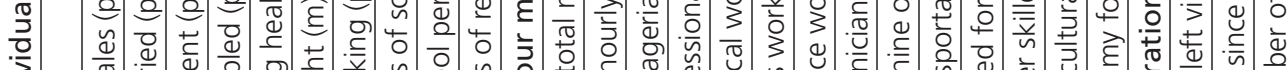

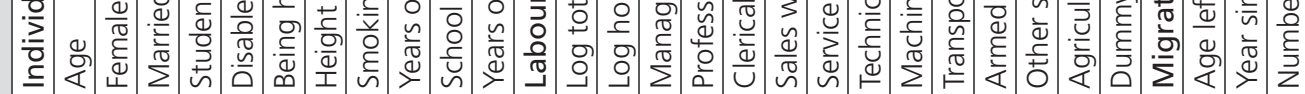




\section{Analyses}

\section{The selection model}

Tables 15.3a and 15.3b show the estimates for Equation 15.2 using the RUMiCI rural sample for China and the combined RUMiCI and Susenas rural sample for Indonesia. As tends to be the case, the relatively young and highly educated are the ones more likely to migrate, as witnessed by the positive coefficient on education and the negative coefficient on age. The results on gender and marital status, however, differ significantly between the two countries. In China, men are 10 per cent more likely to move to cities, while gender plays no role in explaining migration probabilities for Indonesia. In addition, singles are more likely to migrate in China but the opposite is true for the Indonesian case. These differences fit the difference in institutional settings in that in China it is hard to bring children and families into the cities whereas this is not a particular problem in Indonesia.

Tables 15.3c and 15.3d show the selection equations for Indonesia separating recent and lifelong migration. This is mainly to see if it makes much of a difference to the determinants of migration and hence the sensitivity of the later analyses to the definition of migration. If we focus on two key characteristics, years of schooling and the network size (where the network is the eventual instrument), then we see that there is not much difference between the findings of Tables $15.3 \mathrm{~b}$ to $15.3 \mathrm{~d}$. The marginal effect of years of schooling changes from 0.121 in Table $15.3 \mathrm{~b}$ to 0.118 in Table $15.3 \mathrm{c}$ and 0.135 in Table $15.3 \mathrm{~d}$, well within each other's confidence intervals. The effects of the network size is always of the same sign and similar magnitude in each of these three tables, with the effects of the network size in Medan, Tangerang and Samarinda within each other's confidence interval. Only for Makassar is it the case that for more recent migrants, the effect of the network size is significantly smaller (16.8 in Table 15.3c as compared to 20.2 in Table 15.3b and 21.0 IN Table 15.3d) but these effects are still within 20 per cent of each other. 
Table 15.3a Marginal effect from migration selection equation: China

\begin{tabular}{|c|c|c|c|c|c|c|}
\hline & \multicolumn{3}{|c|}{ Probit estimates } & \multicolumn{3}{|c|}{ OLS estimates } \\
\hline & Total & Males & Females & Total & Males & Females \\
\hline \multirow{2}{*}{$\begin{array}{l}\text { Proportion of village labour } \\
\text { force migrated in } 2005\end{array}$} & 0.340 & 0.387 & 0.269 & 0.310 & 0.338 & 0.274 \\
\hline & {$[0.042]^{* * *}$} & {$[0.051]^{* * *}$} & {$[0.036]^{* * *}$} & {$[0.038]^{* * *}$} & {$[0.044]^{* * *}$} & {$[0.038]^{* * *}$} \\
\hline \multirow[t]{2}{*}{ Age } & 0.009 & 0.018 & 0.009 & -0.015 & -0.005 & -0.027 \\
\hline & {$[0.004]^{* *}$} & {$[0.005]^{* * *}$} & {$[0.004]^{* *}$} & {$[0.003]^{* * *}$} & {$[0.004]$} & {$[0.004]^{* * *}$} \\
\hline \multirow[t]{2}{*}{$\mathrm{Age}^{2}$} & -0.035 & -0.049 & -0.034 & 0.001 & -0.013 & 0.019 \\
\hline & {$[0.005]^{* * *}$} & {$[0.007]^{* * *}$} & {$[0.006]^{* * *}$} & {$[0.004]$} & {$[0.005]^{* *}$} & {$[0.005]^{* * *}$} \\
\hline \multirow[t]{2}{*}{ Dummy for males } & 0.109 & & & 0.096 & & \\
\hline & {$[0.010]^{* * *}$} & & & {$[0.009]^{* * *}$} & & \\
\hline \multirow[t]{2}{*}{ Years of schooling } & 0.026 & 0.007 & 0.025 & 0.015 & 0.010 & 0.020 \\
\hline & {$[0.010]^{* *}$} & {$[0.015]$} & {$[0.011]^{* *}$} & {$[0.007]^{* *}$} & {$[0.011]$} & {$[0.008]^{* *}$} \\
\hline \multirow[t]{2}{*}{ Years of schooling ${ }^{2}$} & -0.001 & 0.000 & -0.001 & -0.001 & -0.001 & -0.001 \\
\hline & {$[0.001]^{* *}$} & {$[0.001]$} & {$[0.001]^{*}$} & {$[0.000]^{*}$} & {$[0.001]$} & {$[0.001]^{*}$} \\
\hline \multirow{2}{*}{$\begin{array}{l}\text { School performance (good/ } \\
\text { very good) }\end{array}$} & -0.017 & -0.021 & -0.017 & -0.017 & -0.016 & -0.020 \\
\hline & [0.011] & {$[0.013]$} & {$[0.011]$} & {$[0.010]^{*}$} & {$[0.011]$} & {$[0.012]^{*}$} \\
\hline \multirow[t]{2}{*}{ Healthy and very healthy } & 0.032 & 0.040 & 0.018 & 0.026 & 0.032 & 0.018 \\
\hline & {$[0.014]^{\star *}$} & {$[0.018]^{* *}$} & {$[0.015]$} & {$[0.011]^{* *}$} & {$[0.014]^{* *}$} & {$[0.012]$} \\
\hline \multirow[t]{2}{*}{ Height } & 0.001 & 0.002 & -0.001 & 0.001 & 0.002 & -0.001 \\
\hline & [0.001] & [0.001] & {$[0.001]$} & {$[0.001]$} & {$[0.001]$} & {$[0.001]$} \\
\hline \multirow[t]{2}{*}{ Married } & -0.060 & -0.044 & -0.094 & -0.057 & -0.028 & -0.091 \\
\hline & {$[0.016]^{* \star *}$} & {$[0.021]^{\star *}$} & {$[0.020]^{* * *}$} & {$[0.017]^{* * *}$} & {$[0.020]$} & {$[0.021]^{* * *}$} \\
\hline \multirow{2}{*}{$\begin{array}{l}\text { Number of children ever } \\
\text { given birth to }\end{array}$} & -0.017 & -0.022 & -0.008 & -0.020 & -0.021 & -0.018 \\
\hline & {$[0.007]^{\star *}$} & {$[0.009]^{\star *}$} & [0.008] & {$[0.006]^{* * *}$} & {$[0.007]^{* * *}$} & {$[0.007]^{* * *}$} \\
\hline \multirow[t]{2}{*}{ Birth order } & -0.004 & -0.009 & 0.000 & -0.004 & -0.008 & 0.001 \\
\hline & {$[0.003]$} & {$[0.004]^{* *}$} & {$[0.004]$} & {$[0.002]$} & {$[0.003]^{* *}$} & [0.003] \\
\hline \multirow{2}{*}{$\begin{array}{l}\text { Daily wage for unskilled } \\
\text { workers in the village }\end{array}$} & -0.001 & -0.001 & -0.001 & -0.001 & -0.001 & -0.001 \\
\hline & [0.001] & {$[0.001]$} & [0.001] & {$[0.001]^{*}$} & {$[0.001]^{*}$} & [0.001] \\
\hline \multirow[t]{2}{*}{ Hilly area } & -0.004 & -0.018 & 0.012 & -0.006 & -0.019 & 0.010 \\
\hline & {$[0.020]$} & {$[0.025]$} & {$[0.022]$} & {$[0.018]$} & {$[0.022]$} & {$[0.022]$} \\
\hline \multirow[t]{2}{*}{ Mountainous area } & 0.127 & 0.140 & 0.119 & 0.104 & 0.113 & 0.100 \\
\hline & {$[0.040]^{* * *}$} & {$[0.047]^{* * *}$} & {$[0.045]^{* * *}$} & {$[0.029]^{* * *}$} & {$[0.035]^{* * *}$} & {$[0.034]^{* * *}$} \\
\hline Province dummies & Yes & Yes & Yes & Yes & Yes & Yes \\
\hline F-test of the instrument & 68.63 & 57.84 & 56.99 & 68.17 & 59.05 & 52.73 \\
\hline Observations & 16499 & 9061 & 7438 & 16499 & 9061 & 7438 \\
\hline Adjusted $\mathrm{R}^{2}$ & & & & 0.27 & 0.25 & 0.29 \\
\hline
\end{tabular}

* significant at 10 per cent

** significant at 5 per cent

*** significant at 1 per cent

Note: Robust standard errors in square brackets. 
Table 15.3b Marginal effect from migration selection equation: Indonesia

\begin{tabular}{|c|c|c|c|c|c|c|}
\hline & \multicolumn{3}{|c|}{ Probit estimates } & \multicolumn{3}{|c|}{ OLS estimates } \\
\hline & Total & Males & Females & Total & Males & Females \\
\hline \multirow[t]{2}{*}{ Dummy for females } & 0.062 & & & 0.002 & & \\
\hline & $(0.048)$ & & & $(0.002)$ & & \\
\hline \multirow[t]{2}{*}{ Age } & $-1.901 * * *$ & -0.013 & $-3.935 * * *$ & $-0.045^{* *}$ & 0.002 & $-0.077 * * *$ \\
\hline & $(0.706)$ & $(1.115)$ & $(0.824)$ & $(0.022)$ & $(0.036)$ & $(0.023)$ \\
\hline \multirow[t]{2}{*}{$\mathrm{Age}^{2}$} & $0.000 * * *$ & 0.000 & $0.000 * * *$ & $0.000 * *$ & 0.000 & $0.000 * * *$ \\
\hline & $(0.000)$ & $(0.000)$ & $(0.000)$ & $(0.000)$ & $(0.000)$ & $(0.000)$ \\
\hline \multirow[t]{2}{*}{ Dummy for students } & $-0.764 * * *$ & $-0.427 * * *$ & $-1.005^{* * *}$ & $-0.014^{* * *}$ & $-0.011 * * *$ & $-0.017 * * *$ \\
\hline & $(0.059)$ & $(0.085)$ & $(0.082)$ & $(0.001)$ & $(0.003)$ & $(0.002)$ \\
\hline \multirow[t]{2}{*}{ Years of schooling } & $0.121 * * *$ & $0.151 * * *$ & $0.094 * * *$ & 0.001 & 0.001 & 0.001 \\
\hline & $(0.026)$ & $(0.040)$ & $(0.033)$ & $(0.001)$ & $(0.001)$ & $(0.001)$ \\
\hline \multirow[t]{2}{*}{ Years of schooling ${ }^{2}$} & $-0.002 *$ & $-0.003^{*}$ & -0.001 & $0.000 * *$ & $0.000 *$ & 0.000 \\
\hline & $(0.001)$ & $(0.002)$ & $(0.001)$ & $(0.000)$ & $(0.000)$ & $(0.000)$ \\
\hline \multirow[t]{2}{*}{ Married } & $0.218 * * *$ & 0.118 & $0.353^{* * *}$ & $0.008 * * *$ & 0.003 & $0.011 * * *$ \\
\hline & $(0.068)$ & $(0.114)$ & $(0.066)$ & $(0.002)$ & $(0.005)$ & $(0.002)$ \\
\hline \multirow[t]{2}{*}{ Size of network in Medan } & $5.943 * * *$ & $6.077 * * *$ & $5.909 * * *$ & $0.835^{* * *}$ & $0.958 * * *$ & $0.712 * *$ \\
\hline & $(0.597)$ & $(0.745)$ & $(0.956)$ & $(0.219)$ & $(0.295)$ & $(0.322)$ \\
\hline \multirow{2}{*}{$\begin{array}{l}\text { Size of network in } \\
\text { Tangerang }\end{array}$} & $27.195^{* * *}$ & $23.186 * * *$ & $32.654^{* * *}$ & $0.920 * * *$ & $0.813^{* *}$ & $1.038 * * *$ \\
\hline & $(5.000)$ & $(7.006)$ & $(6.995)$ & $(0.271)$ & $(0.371)$ & $(0.394)$ \\
\hline \multirow{2}{*}{$\begin{array}{l}\text { Size of network in } \\
\text { Samarinda }\end{array}$} & $19.131 * * *$ & $17.858 * * \star$ & $21.247 * \star *$ & $0.898 * * *$ & $0.879 * * *$ & $0.921 * * *$ \\
\hline & $(1.875)$ & $(2.812)$ & $(2.248)$ & $(0.157)$ & $(0.224)$ & $(0.215)$ \\
\hline \multirow[t]{2}{*}{ Size of network in Makassar } & $20.167 * * *$ & $20.797 * * *$ & $20.128 * * *$ & $1.170 * * *$ & $1.310 * * *$ & $1.025 * * *$ \\
\hline & $(1.715)$ & $(2.874)$ & $(1.555)$ & $(0.143)$ & $(0.245)$ & $(0.135)$ \\
\hline $\begin{array}{l}\text { Dummies for island of } \\
\text { origin }\end{array}$ & Yes & Yes & Yes & Yes & Yes & Yes \\
\hline \multirow[t]{2}{*}{ Constant } & $-3.458 * * *$ & $-4.012 * * *$ & $-2.874 * * *$ & -0.009 & $-0.022 * *$ & 0.002 \\
\hline & (0.229) & $(0.347)$ & $(0.283)$ & $(0.006)$ & $(0.009)$ & $(0.007)$ \\
\hline F-test of the instrument & 392.11 & 185.45 & 285.1 & 42.48 & 20.21 & 29.33 \\
\hline Observations & 70968 & 36622 & 34346 & 70968 & 36622 & 34346 \\
\hline Adjusted $R^{2}$ & & & & 0.090 & 0.100 & 0.082 \\
\hline
\end{tabular}

* significant at 10 per cent

** significant at 5 per cent

*** significant at 1 per cent

Note: Standard errors in parentheses. 
Table 15.3c Marginal effect from recent migration selection equation: Indonesia

\begin{tabular}{|c|c|c|c|c|c|c|}
\hline & \multicolumn{3}{|c|}{ Probit estimates } & \multicolumn{3}{|c|}{ OLS estimates } \\
\hline & Total & Males & Females & Total & Males & Females \\
\hline \multirow[t]{2}{*}{ Dummy for females } & 0.029 & & & 0.003 & & \\
\hline & $(0.080)$ & & & $(0.002)$ & & \\
\hline \multirow[t]{2}{*}{ Age } & $-8.502 * * *$ & $-9.086 * * *$ & $-8.238 * * *$ & $-0.067 * \star *$ & $-0.090 * * *$ & $-0.049 * *$ \\
\hline & $(1.239)$ & $(1.369)$ & $(1.891)$ & $(0.014)$ & $(0.024)$ & $(0.024)$ \\
\hline \multirow[t]{2}{*}{$\mathrm{Age}^{2}$} & $0.001 * * *$ & $0.001 * * *$ & $0.001 * * *$ & $0.000 * * *$ & $0.000 * * *$ & $0.000 * *$ \\
\hline & $(0.000)$ & $(0.000)$ & $(0.000)$ & $(0.000)$ & $(0.000)$ & $(0.000)$ \\
\hline \multirow[t]{2}{*}{ Dummy for students } & $-0.402 * * *$ & -0.040 & $-0.649 * * *$ & $-0.005^{* *}$ & -0.000 & $-0.007 * *$ \\
\hline & $(0.077)$ & $(0.076)$ & $(0.110)$ & $(0.002)$ & $(0.001)$ & $(0.003)$ \\
\hline \multirow[t]{2}{*}{ Years of schooling } & $0.118^{*}$ & 0.068 & 0.152 & -0.000 & -0.001 & 0.001 \\
\hline & $(0.060)$ & $(0.056)$ & $(0.093)$ & $(0.001)$ & $(0.001)$ & $(0.001)$ \\
\hline \multirow[t]{2}{*}{ Years of schooling ${ }^{2}$} & -0.002 & 0.001 & -0.003 & $0.000^{*}$ & $0.000 *$ & 0.000 \\
\hline & $(0.003)$ & $(0.002)$ & $(0.004)$ & $(0.000)$ & $(0.000)$ & $(0.000)$ \\
\hline \multirow[t]{2}{*}{ Married } & $0.653^{* * *}$ & $0.842 * * *$ & $0.511 * * *$ & $0.007 * * *$ & $0.008 * * *$ & $0.007 * *$ \\
\hline & $(0.078)$ & $(0.110)$ & $(0.113)$ & $(0.002)$ & $(0.002)$ & $(0.004)$ \\
\hline \multirow[t]{2}{*}{ Size of network in Medan } & $5.177 * * *$ & $1.587 * * *$ & $6.329 * * *$ & 0.459 & 0.007 & 0.766 \\
\hline & $(1.392)$ & $(0.487)$ & $(1.550)$ & $(0.374)$ & $(0.015)$ & $(0.541)$ \\
\hline \multirow{2}{*}{$\begin{array}{l}\text { Size of network in } \\
\text { Tangerang }\end{array}$} & $29.816^{* * *}$ & $21.026^{* * *}$ & $37.696^{* * *}$ & $0.259 * \star$ & 0.109 & $0.371^{* \star}$ \\
\hline & $(4.923)$ & $(5.339)$ & $(7.367)$ & $(0.106)$ & $(0.086)$ & $(0.148)$ \\
\hline \multirow{2}{*}{$\begin{array}{l}\text { Size of network in } \\
\text { Samarinda }\end{array}$} & $19.809 * * *$ & $19.142 * * *$ & $22.352 * * *$ & $0.104 * * *$ & $0.112 * *$ & 0.101 \\
\hline & $(2.041)$ & $(2.674)$ & $(3.252)$ & $(0.040)$ & $(0.044)$ & $(0.065)$ \\
\hline \multirow[t]{2}{*}{ Size of network in Makassar } & $16.822^{* * *}$ & $14.731 * * *$ & $19.572^{* * *}$ & $0.228 * * *$ & $0.284^{* * *}$ & $0.209^{*}$ \\
\hline & $(1.262)$ & $(1.700)$ & $(1.938)$ & $(0.089)$ & $(0.085)$ & $(0.123)$ \\
\hline $\begin{array}{l}\text { Dummies for island of } \\
\text { origin }\end{array}$ & Yes & Yes & Yes & Yes & Yes & Yes \\
\hline \multirow[t]{2}{*}{ Constant } & $-2.403 * * *$ & $-2.106 * * *$ & $-2.534^{* * *}$ & 0.008 & $0.016^{* * *}$ & 0.003 \\
\hline & $(0.503)$ & $(0.410)$ & $(0.734)$ & $(0.007)$ & $(0.005)$ & $(0.010)$ \\
\hline F-test of the instrument & 315.6 & 176.45 & 170.25 & 15.12 & 10.1 & 8.89 \\
\hline Observations & 69475 & 35831 & 33644 & 69401 & 35793 & 33608 \\
\hline Adjusted $\mathrm{R}^{2}$ & & & & 0.065 & 0.010 & 0.156 \\
\hline
\end{tabular}

* significant at 10 per cent

** significant at 5 per cent

*** significant at 1 per cent

Note: Standard errors in parentheses. 
Table 15.3d Marginal effect from lifetime migration selection equation: Indonesia

\begin{tabular}{|c|c|c|c|c|c|c|}
\hline & \multicolumn{3}{|c|}{ Probit estimates } & \multicolumn{3}{|c|}{ OLS estimates } \\
\hline & Total & Males & Females & Total & Males & Females \\
\hline \multirow[t]{2}{*}{ Dummy for females } & 0.090 & & & 0.002 & & \\
\hline & $(0.059)$ & & & $(0.011)$ & & \\
\hline \multirow[t]{2}{*}{ Age } & $3.787 * * *$ & $7.201 * * *$ & 0.572 & 0.005 & 0.046 & 0.004 \\
\hline & $(1.009)$ & $(1.753)$ & $(1.099)$ & $(0.246)$ & $(0.491)$ & $(0.203)$ \\
\hline \multirow[t]{2}{*}{$\mathrm{Age}^{2}$} & $-0.000 * *$ & $-0.001 * * *$ & 0.000 & 0.000 & 0.000 & 0.000 \\
\hline & $(0.000)$ & $(0.000)$ & $(0.000)$ & $(0.000)$ & $(0.000)$ & $(0.000)$ \\
\hline \multirow[t]{2}{*}{ Dummy for students } & $-1.480 * * *$ & $-1.112 * * *$ & $-1.705^{* * *}$ & $-0.040 * * *$ & $-0.037 * *$ & $-0.040 * * *$ \\
\hline & $(0.147)$ & $(0.177)$ & $(0.230)$ & $(0.008)$ & $(0.017)$ & $(0.008)$ \\
\hline \multirow[t]{2}{*}{ Years of schooling } & $0.135^{* * *}$ & $0.177^{* * *}$ & $0.095^{* * *}$ & 0.003 & 0.002 & 0.003 \\
\hline & $(0.030)$ & $(0.048)$ & $(0.035)$ & $(0.006)$ & $(0.010)$ & $(0.005)$ \\
\hline \multirow[t]{2}{*}{ Years of schooling ${ }^{2}$} & $-0.003 * \star$ & $-0.004 * *$ & -0.002 & 0.000 & 0.000 & 0.000 \\
\hline & $(0.001)$ & $(0.002)$ & $(0.002)$ & $(0.000)$ & $(0.001)$ & $(0.000)$ \\
\hline \multirow[t]{2}{*}{ Married } & 0.007 & $-0.270 * *$ & $0.306^{* * *}$ & 0.010 & 0.004 & 0.014 \\
\hline & $(0.082)$ & $(0.132)$ & $(0.084)$ & $(0.016)$ & $(0.036)$ & $(0.017)$ \\
\hline \multirow{2}{*}{$\begin{array}{l}\text { Proportion of those in the } \\
\text { same rural district migrated } \\
\text { to Medan }\end{array}$} & $6.188^{* * *}$ & $6.397 * * *$ & $5.830 * * *$ & $0.271 *$ & $0.436 * *$ & 0.097 \\
\hline & $(0.673)$ & $(0.756)$ & $(1.277)$ & $(0.148)$ & $(0.222)$ & $(0.187)$ \\
\hline \multirow{2}{*}{$\begin{array}{l}\text { Proportion of those in the } \\
\text { same rural district migrated } \\
\text { to Tangerang }\end{array}$} & $24.768 * * *$ & $21.914 * *$ & $28.637 * * *$ & 1.137 & -0.060 & $2.452 * * *$ \\
\hline & $(6.807)$ & (9.194) & (9.966) & $(1.141)$ & $(2.027)$ & $(0.672)$ \\
\hline \multirow{2}{*}{$\begin{array}{l}\text { Proportion of those in the } \\
\text { same rural district migrated } \\
\text { to Samarinda }\end{array}$} & $16.548 * * *$ & $15.552 * * *$ & $17.940 * * *$ & -0.197 & 0.370 & -0.776 \\
\hline & $(2.556)$ & $(4.068)$ & $(2.633)$ & $(0.595)$ & $(0.729)$ & $(0.924)$ \\
\hline \multirow{2}{*}{$\begin{array}{l}\text { Proportion of those in the } \\
\text { same rural district migrated } \\
\text { to Makassar }\end{array}$} & $21.001 * * *$ & $23.617 * * *$ & $18.988 * * *$ & $-1.297 * \star$ & $-1.855^{\star}$ & -0.715 \\
\hline & $(2.432)$ & $(4.160)$ & $(1.943)$ & $(0.652)$ & $(0.964)$ & $(0.850)$ \\
\hline $\begin{array}{l}\text { Dummies for island of } \\
\text { origin }\end{array}$ & Yes & Yes & Yes & Yes & Yes & Yes \\
\hline \multirow[t]{2}{*}{ Constant } & $-4.959 * * *$ & $-5.848 * * *$ & $-4.107 * * *$ & -0.036 & -0.038 & -0.039 \\
\hline & $(0.300)$ & $(0.481)$ & $(0.363)$ & $(0.061)$ & $(0.115)$ & $(0.042)$ \\
\hline F-test of the instrument & 228.69 & 128.6 & 168.08 & 2.46 & 1.76 & 4.87 \\
\hline Observations & 70164 & 36204 & 33960 & 70177 & 36210 & 33967 \\
\hline Adjusted $\mathrm{R}^{2}$ & & & & 0.046 & 0.034 & 0.076 \\
\hline
\end{tabular}

* significant at 10 per cent

** significant at 5 per cent

*** significant at 1 per cent

Note: Standard errors in parentheses. 
For the Chinese case, we show the extended selection estimations, which control for health, height, birth order and village-level information. Individuals who are healthier, with fewer children, and from mountainous areas (which tend to be poorer) are more likely to migrate. This information is not available in the Indonesian rural data, but, as a robustness check, we redid all the Chinese estimations using the same selection specification as we used in the Indonesian estimations, which did not significantly change the main results. ${ }^{4}$

The instrument used to identify the migration selection equation in the case of China is the proportion of the labour force migrated from the migrant's village in 2005. For Indonesia, we use the proportion of the labour force in each migrant's rural district migrating to the cities included in the survey - that is, Medan, Tangerang, Samarinda or Makassar - in 2007-08. In both countries, we find that the instrument is highly significant and of the expected sign: the higher the proportion who previously migrated to a particular destination, the more likely someone from that region is to migrate in 2008. The F-test presented at the bottom of each table signals that there is no weak instrument problem.

The superior fit of the estimation results in China is probably related to the greater wage differential between the cities and the rural countryside, as well as to the fact that migration is mainly temporary; everyone who can profitably migrate in China does so, at least for a few years, and our controls seem to pick up the factors that predict this profitability of migration. In Indonesia, other unmeasured factors presumably prevent individuals from migrating (which is a more permanent choice in Indonesia).

Using the estimated results from Tables 15.3a and 15.3b, we predict the probability of migration for the RUMiCI urban migrant samples; we calculate 7 for each individual, which we include in the earnings equation as a Heckman correction term. The predicted migration probability is plotted for migrants and the rural samples (see Figure 15.3). The figures show that the predicted probability and 7 for the sample of migrants observed in cities (out-ofsample predictions) exhibit almost the same pattern as those observed for the migrants in the rural household sample (in-sample predictions), indicating the similarity of the migrants observed from the two samples. 
Figure 15.3 Predicted probability of migration: China

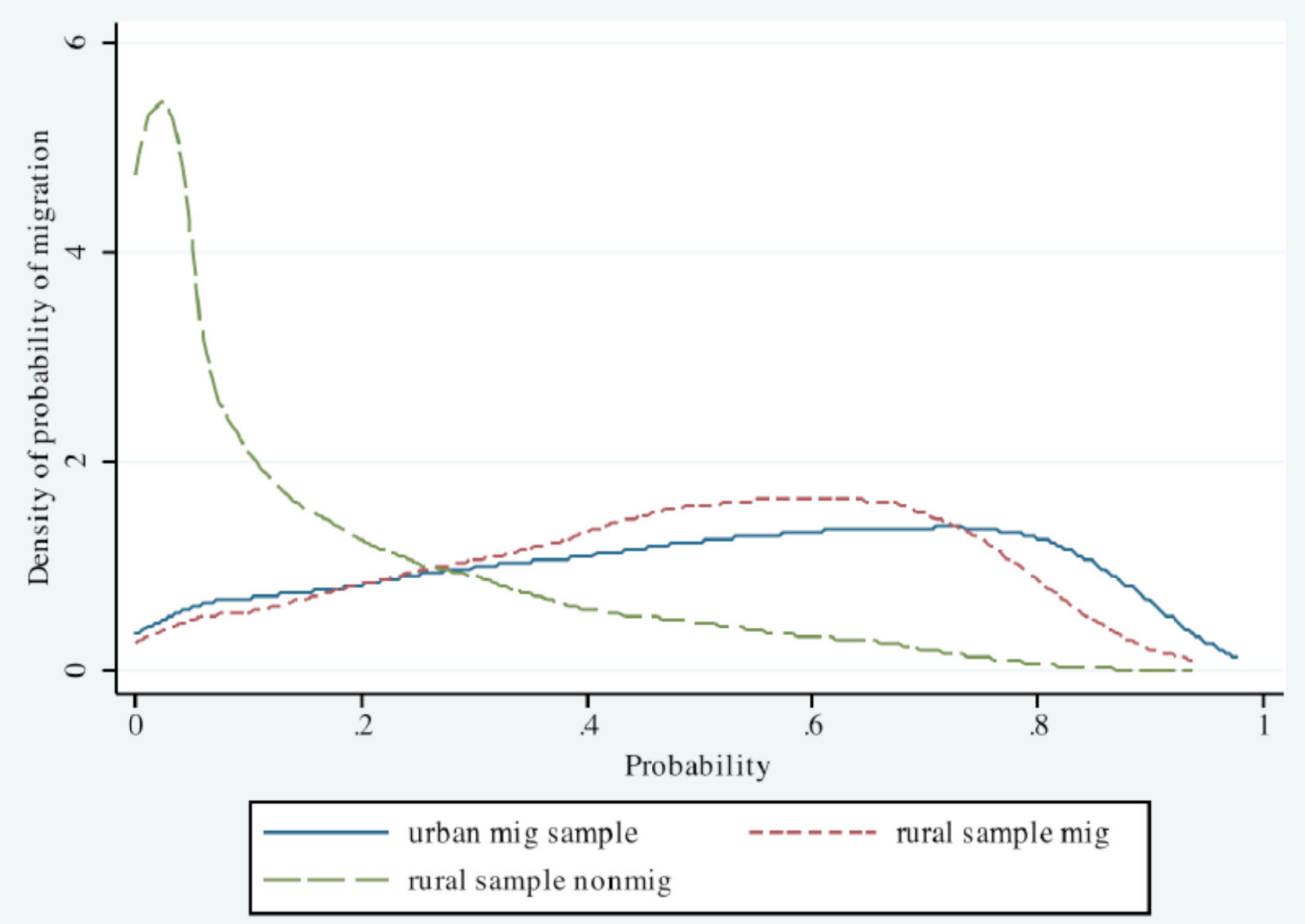

\section{The earnings model}

The main wage regression results are reported in Tables 15.4a and 15.4b. The two columns of each table show the progression from not correcting for selectivity (simple OLS) to the inclusion of a selection correction term. For both countries, we provide results for hourly earnings from the main job. We see in column 1 of Table 15.4a that the effect of being a migrant in China - comparable with being a recent migrant in Indonesia - is to have nearly 56 per cent lower hourly wages than their urban incumbents. This does not yet allow for selection. The equivalent result in Table 15.4b for Indonesia is that a recent migrant earns nearly 17.5 per cent more than their urban incumbents. Given that migrants work more hours, total monthly earnings of recent migrants are about 30 per cent higher than the earnings of corresponding urban workers (Table 15.4c). 
Table 15.4a Results from the earnings equations (without occupation)

\begin{tabular}{|c|c|c|c|c|}
\hline & OLS & $\begin{array}{l}\text { OLS with } \\
\text { lambda }\end{array}$ & $\begin{array}{l}\text { IV with } \\
\text { predicted } \\
\text { probability } \\
\text { using OLS }\end{array}$ & $\begin{array}{c}\text { IV with } \\
\text { predicted } \\
\text { probability } \\
\text { using probit }\end{array}$ \\
\hline \multirow[t]{2}{*}{ Dummy for migrants } & -0.560 & -0.914 & -0.646 & -0.463 \\
\hline & {$[0.086]^{* * *}$} & {$[0.119]^{* * *}$} & {$[0.081]^{* * *}$} & {$[0.067]^{* * *}$} \\
\hline \multirow[t]{2}{*}{ Lambda } & & 0.965 & & \\
\hline & & {$[0.225]^{* * *}$} & & \\
\hline \multirow[t]{2}{*}{ Age } & 0.038 & 0.039 & 0.041 & 0.052 \\
\hline & {$[0.007]^{* * *}$} & {$[0.007]^{* * *}$} & {$[0.006]^{* * *}$} & {$[0.006]^{* * *}$} \\
\hline \multirow[t]{2}{*}{$\mathrm{Age}^{2}$} & -0.000 & -0.001 & -0.001 & -0.001 \\
\hline & {$[0.000]^{* * *}$} & {$[0.000]^{* * *}$} & {$[0.000]^{* * *}$} & {$[0.000]^{* * *}$} \\
\hline \multirow[t]{2}{*}{ Males } & 0.112 & 0.139 & 0.148 & 0.145 \\
\hline & {$[0.018]^{* * *}$} & {$[0.019]^{* * *}$} & {$[0.019]^{* * *}$} & {$[0.019]^{* \star *}$} \\
\hline \multirow[t]{2}{*}{ Years of schooling } & 0.058 & 0.065 & 0.067 & 0.069 \\
\hline & {$[0.014]^{* * *}$} & {$[0.014]^{* * *}$} & {$[0.014]^{* * *}$} & {$[0.014]^{* \star *}$} \\
\hline \multirow[t]{2}{*}{ Years of schooling ${ }^{2}$} & -0.001 & -0.002 & -0.002 & -0.002 \\
\hline & {$[0.001]^{*}$} & {$[0.001]^{* *}$} & {$[0.001]^{* *}$} & {$[0.001]^{* *}$} \\
\hline \multirow[t]{2}{*}{ Good school performance } & 0.130 & 0.125 & 0.124 & 0.126 \\
\hline & {$[0.013]^{* * *}$} & {$[0.013]^{* * *}$} & {$[0.013]^{* * *}$} & {$[0.013]^{* \star *}$} \\
\hline \multirow{2}{*}{$\begin{array}{l}\text { National college } \\
\text { examination score }\end{array}$} & 0.001 & 0.001 & 0.001 & 0.001 \\
\hline & {$[0.000]^{* * *}$} & {$[0.000]^{* * *}$} & {$[0.000]^{* * *}$} & {$[0.000]^{* * *}$} \\
\hline \multirow[t]{2}{*}{ Healthy } & 0.050 & 0.056 & 0.058 & 0.060 \\
\hline & {$[0.015]^{* * *}$} & {$[0.015]^{* * *}$} & {$[0.015]^{* * *}$} & {$[0.015]^{* \star *}$} \\
\hline \multirow[t]{2}{*}{ Height } & 0.008 & 0.008 & 0.008 & 0.008 \\
\hline & {$[0.001]^{* * *}$} & {$[0.001]^{* * *}$} & {$[0.001]^{* * *}$} & {$[0.001]^{* \star *}$} \\
\hline \multirow[t]{2}{*}{ Married } & 0.116 & 0.090 & 0.084 & 0.101 \\
\hline & {$[0.023]^{* * *}$} & {$[0.024]^{* * *}$} & {$[0.024]^{* * *}$} & {$[0.024]^{* * *}$} \\
\hline \multirow[t]{2}{*}{ Number of children } & -0.081 & -0.088 & -0.088 & -0.085 \\
\hline & {$[0.014]^{* * *}$} & {$[0.014]^{* * *}$} & {$[0.014]^{* * *}$} & {$[0.014]^{* * *}$} \\
\hline \multirow[t]{2}{*}{ Birth order } & -0.015 & -0.016 & -0.017 & -0.016 \\
\hline & {$[0.006]^{* *}$} & {$[0.006]^{* * *}$} & {$[0.006]^{* * *}$} & {$[0.006]^{* * *}$} \\
\hline City dummies & Yes & Yes & Yes & Yes \\
\hline \multicolumn{5}{|c|}{ Migrant specific information } \\
\hline \multirow[t]{2}{*}{ Age left home village } & -0.005 & -0.013 & -0.016 & -0.018 \\
\hline & {$[0.002]^{* * *}$} & {$[0.003]^{* * *}$} & {$[0.001]^{* * *}$} & {$[0.001]^{* * *}$} \\
\hline \multirow[t]{2}{*}{ Years since migration } & 0.017 & 0.011 & 0.007 & 0.005 \\
\hline & {$[0.005]^{* * *}$} & {$[0.005]^{* *}$} & {$[0.004]$} & {$[0.004]$} \\
\hline \multirow[t]{2}{*}{ Years since migration ${ }^{2}$} & -0.001 & -0.001 & -0.000 & -0.001 \\
\hline & {$[0.000]^{* * *}$} & {$[0.000]^{* * *}$} & {$[0.000]^{* * *}$} & {$[0.000]^{* * *}$} \\
\hline
\end{tabular}




\begin{tabular}{|c|c|c|c|c|}
\hline & OLS & $\begin{array}{l}\text { OLS with } \\
\text { lambda }\end{array}$ & $\begin{array}{l}\text { IV with } \\
\text { predicted } \\
\text { probability } \\
\text { using OLS }\end{array}$ & $\begin{array}{c}\text { IV with } \\
\text { predicted } \\
\text { probability } \\
\text { using probit }\end{array}$ \\
\hline \multicolumn{5}{|l|}{ Village information } \\
\hline \multirow{2}{*}{$\begin{array}{l}\text { Daily wage for unskilled in } \\
\text { home village }\end{array}$} & 0.002 & 0.002 & 0.001 & 0.001 \\
\hline & {$[0.001]^{* * *}$} & {$[0.001]^{\star *}$} & {$[0.001]^{* *}$} & {$[0.001]^{*}$} \\
\hline \multirow[t]{2}{*}{ Home village in hilly area } & -0.007 & -0.006 & -0.006 & -0.005 \\
\hline & {$[0.022]$} & {$[0.022]$} & {$[0.022]$} & {$[0.022]$} \\
\hline \multirow{2}{*}{$\begin{array}{l}\text { Home village in } \\
\text { mountainous area }\end{array}$} & -0.037 & 0.017 & 0.033 & 0.018 \\
\hline & {$[0.022]^{*}$} & {$[0.025]$} & {$[0.024]$} & {$[0.024]$} \\
\hline Home town province & Yes & Yes & Yes & Yes \\
\hline Observations & 8950 & 8950 & 8950 & 8950 \\
\hline R-squared & 0.41 & 0.41 & 0.41 & 0.41 \\
\hline
\end{tabular}

* significant at 10 per cent

** significant at 5 per cent

*** significant at 1 per cent

Note: Standard errors in square brackets. 


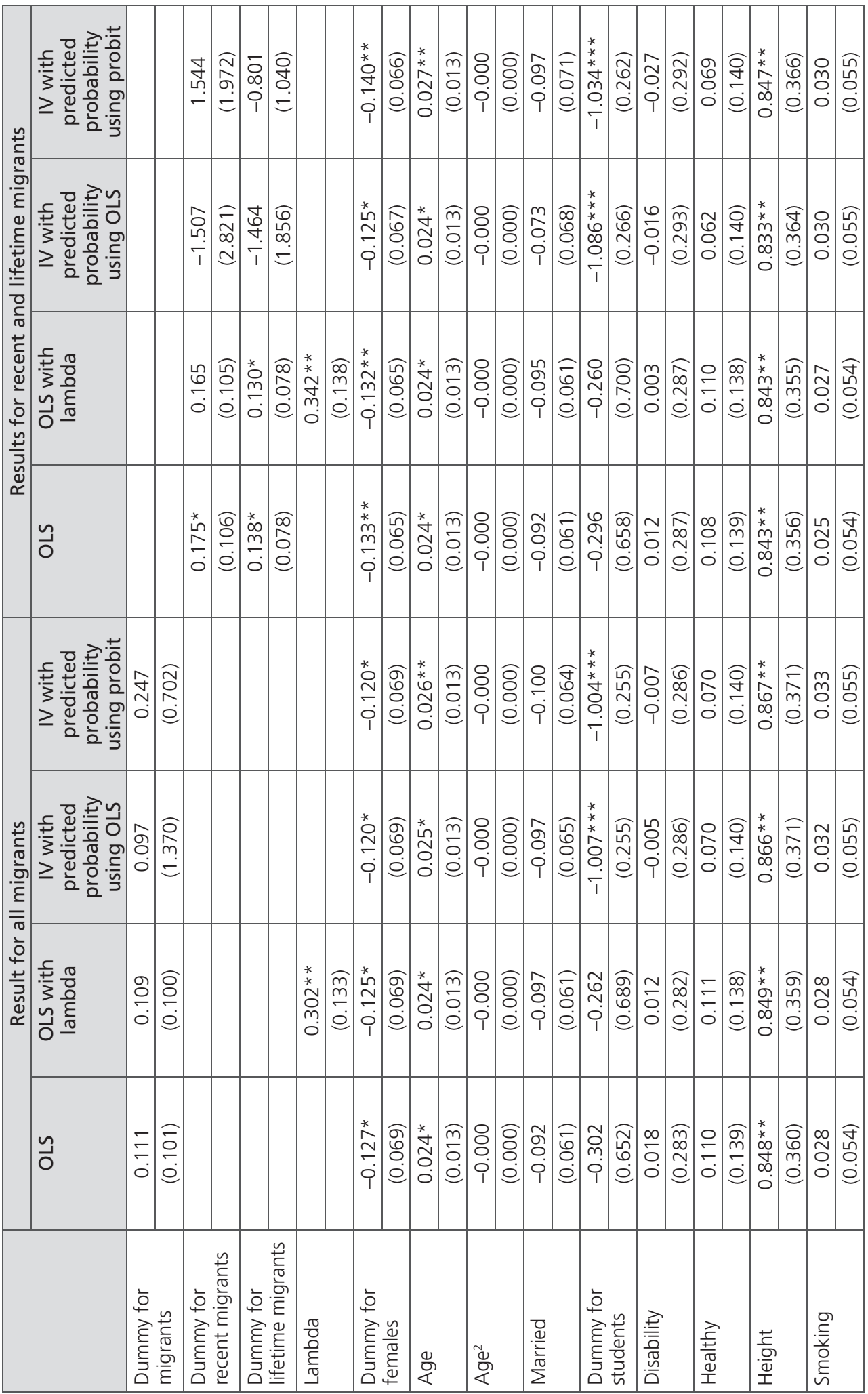




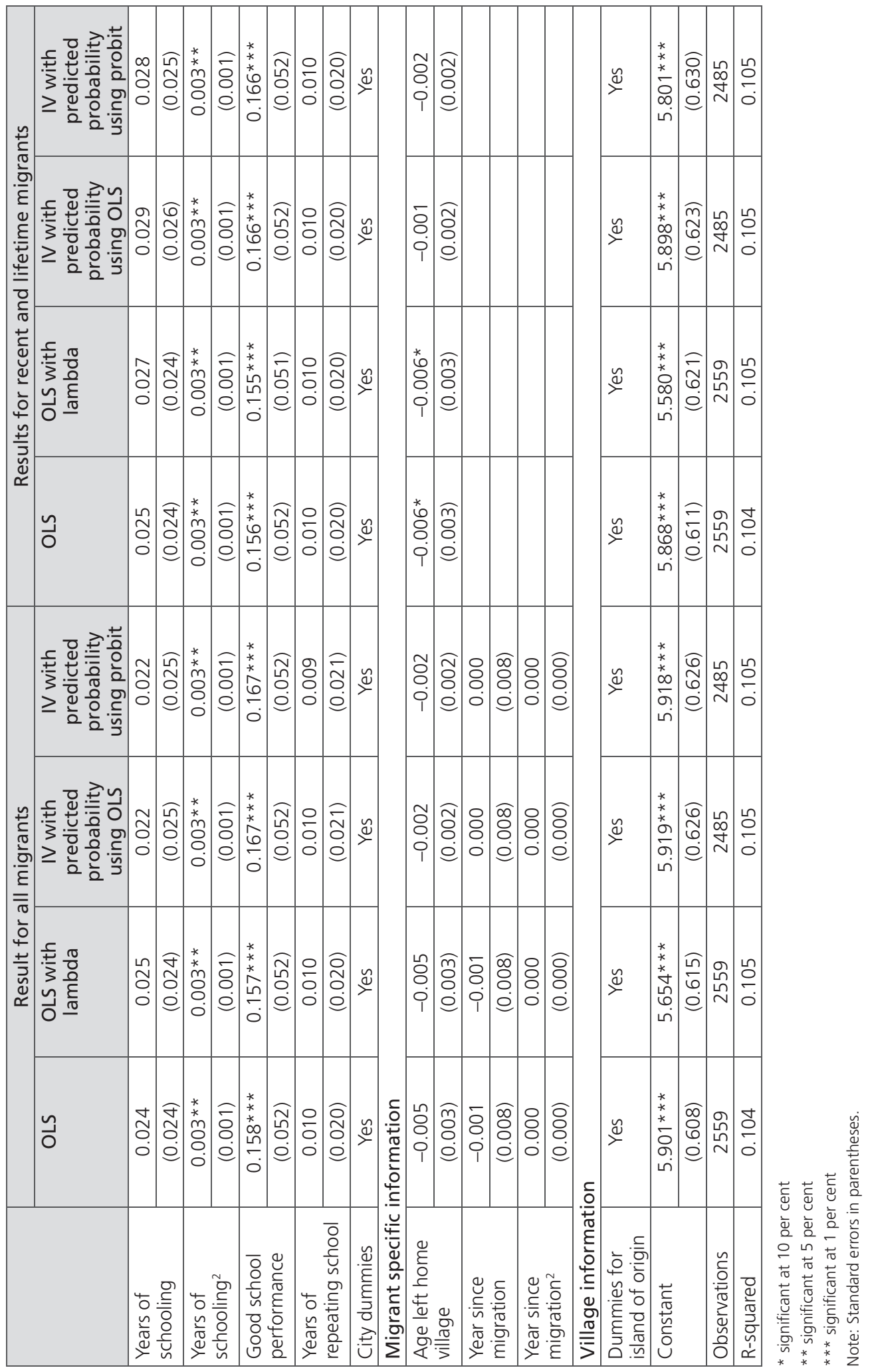




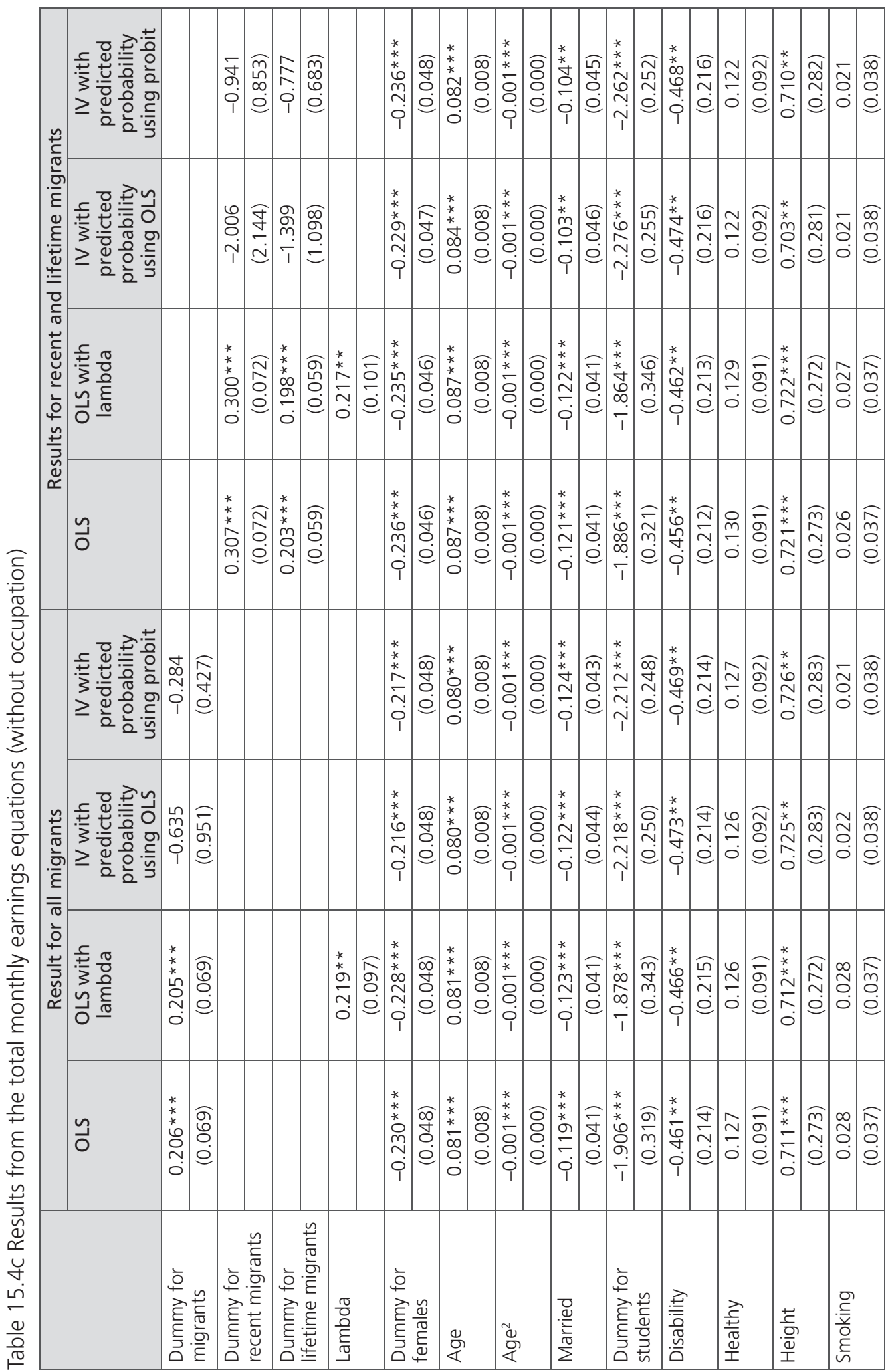




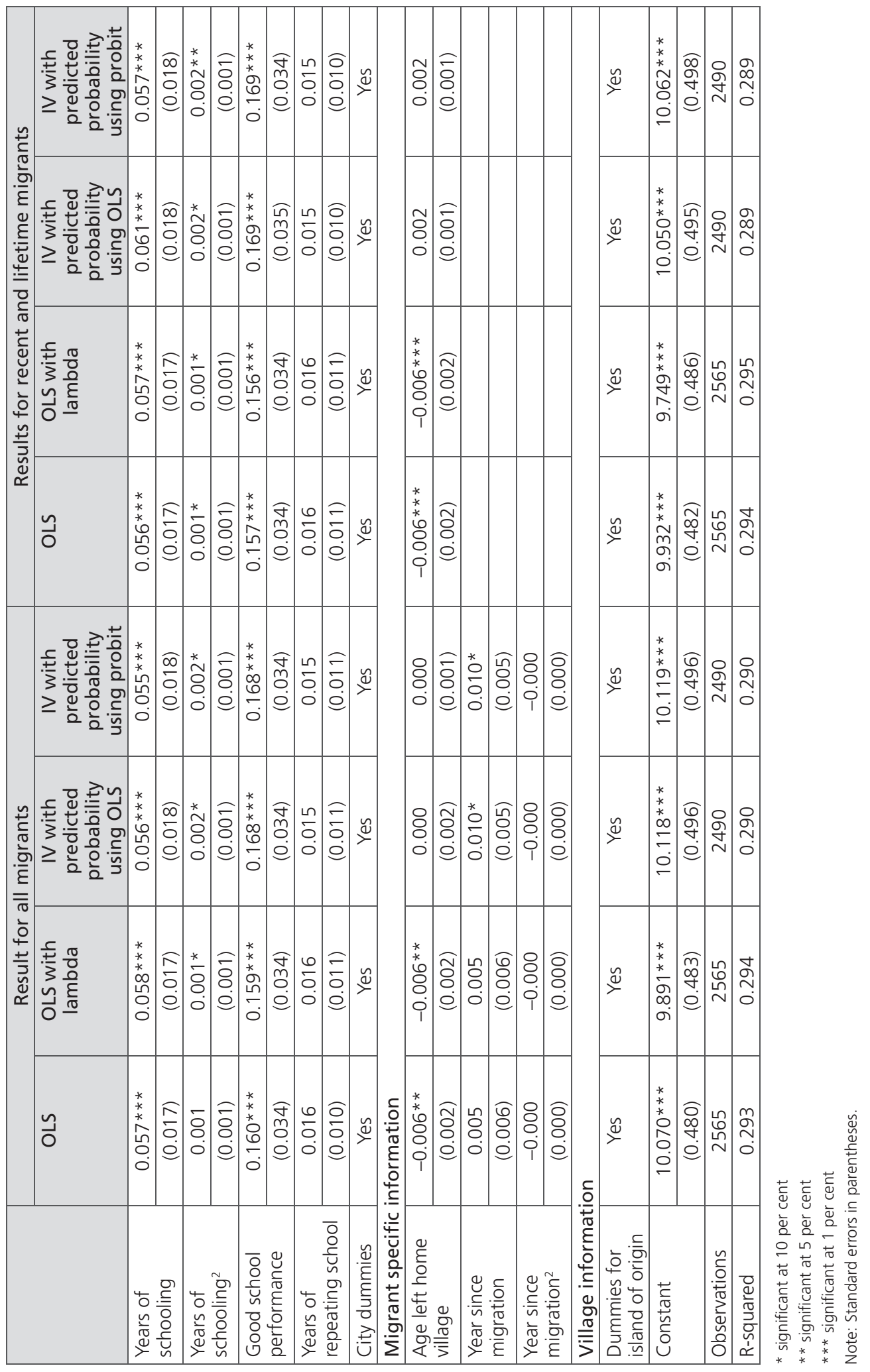


When we then add selection effects (column 2 in each table), we find that in both countries the point estimate of $\bar{\lambda}_{i}$ is positive, indicating positive self-selection of migrants. This selection effect is highly significant in China but insignificant in Indonesia, which reiterates the poorer fit in terms of explaining migration in Indonesia. The inclusion of selection decreases the effect of being a migrant in both countries; migrants in China are now predicted to earn 0.914 log-points less than their urban counterparts whilst in Indonesia the point estimate has become insignificantly negative. For the Chinese case, (the unobservable selection effect) contributes to a 35 per cent increase in earnings for migrants (which is the change in the migrant dummy when not allowing for selection). In Indonesia, this is approximately 1 per cent.

The effect of the other variables on wages is as expected for both countries. For China, the age-earnings profile is inverse-U shaped; the more educated earn more, as do those whose high-school performance was self-rated as being good or very good. To better control for observed ability, we also include the self-reported National College Entrance Examination (NCEE) score variable, which is set to zero if the individual did not take the NCEE. We find that the test score is positively and highly significantly related to one's earnings. In addition, healthy and taller people earn more, which is consistent with findings from other countries. Men earn more, as do individuals who are married, whereas individuals who have more children and are of higher birth order earn less.

Consistent with the international migration literature, we find that individuals who left their village at younger ages earn more, as do individuals who migrated longer ago. One interpretation is that this positive affect of having been somewhere for a long time is an indication of migrants 'catching up' with the locals because of familiarising themselves with local labour-market knowledge and language and eventually reaching similar levels of local human-capital variables.

The regressions also include each migrant's home village characteristics, including the daily wage for unskilled workers and geographic location. We find that individuals who come from villages where wages are higher earn more. Whether or not the hometown is in a mountainous area does not appear to matter.

In the case of Indonesia, we find similar results as in China regarding the effect of individual characteristics: the age-earnings profile is inverse-U shaped; the more educated earn more, as do those whose high-school performance is self-rated as being good or very good. Healthier and taller people earn more, men earn more, those still in school earn less, and those who left their village at a younger age earn more.

The earnings equations estimated above for the two countries suggest that migrants in China are paid extremely low earnings relative to their urban incumbent counterparts, whereas in Indonesia migrants are paid equal earnings.

Next we examine the channels through which migrants in China are discriminated against. As discussed in the background section, discrimination in China is believed to function partially via work restrictions on migrants in particular jobs. In Indonesia, such restrictions do not generally exist. This is almost self-evident from the proportion of workers in the major categories - while in China the proportions of migrants and urban locals working as professionals are 1 and 24 per cent, respectively; the same comparison in 
Indonesia is 8 per cent for both migrants and locals. Hence while in China professional jobs (such as those in the civil service) are reserved for urban residents, in Indonesia, they are not.

When adding broad occupational structure in Tables 15.5a and 15.5b in order to absorb this channel for discrimination, we find that in China (Table 15.5a) the effect of migration on wages increases by 12 percentage points from -0.56 to -0.44 (see column 2 of Tables 15.4a and 15.5a). Hence occupational barriers are able to explain about 20 per cent of the wage differences between migrants and city incumbents. In Indonesia (Table 15.5b), the inclusion of the occupational variables makes the selection effect all but disappear and reduces the effect of being a recent migrant to an insignificant 14 per cent. This means that the slightly higher hourly wages of migrants are explained mainly by their over-representation in higher-paying occupations, which can be due either to some unmeasured greater degree of effort or to a compensating wage differential for working in less pleasant occupations. Table 15.1 supports the idea of higher work effort in the sense that migrants also work significantly longer hours. Table 15.5c, which shows the effects of migration when looking at total monthly earnings, show that recent migrants earn a significant 27 per cent more than urban residents, mainly because of the longer hours worked. Again, selection effects are unimportant in Indonesia for recent migrants. 


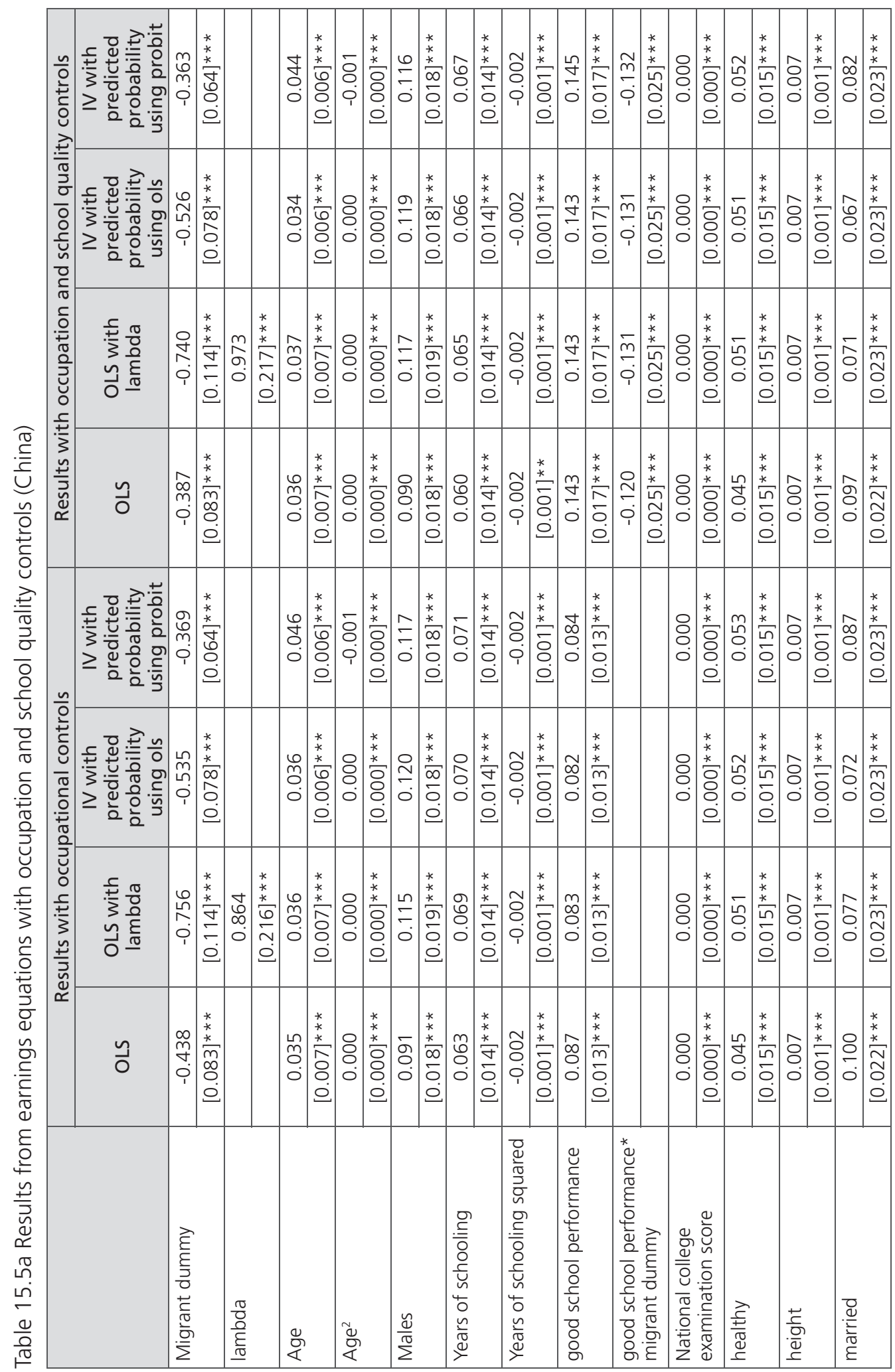




\begin{tabular}{|c|c|c|c|c|c|c|c|c|c|c|c|c|c|c|c|c|c|c|c|}
\hline ऽ & 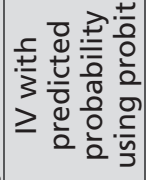 & $\mid \begin{array}{c}0 \\
0 \\
0 \\
0 \\
1\end{array}$ & 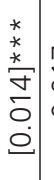 & 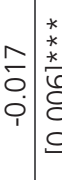 & 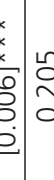 & 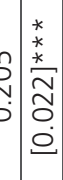 & 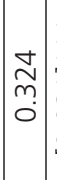 & 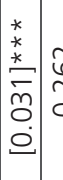 & 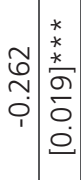 & 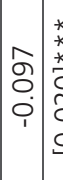 & 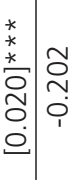 & $\mid$\begin{tabular}{c|c}
$*$ & \\
$*$ & \\
$*$ & \\
$m$ & $n$ \\
$m$ & $\vdots$ \\
0 \\
0 \\
0
\end{tabular} & 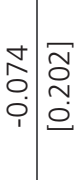 & $\stackrel{\Perp}{=}$ & & 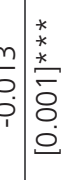 & $\begin{array}{l}\infty \\
0 \\
0 \\
0 \\
\end{array}$ & 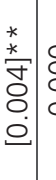 & 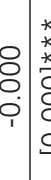 \\
\hline ్ㅡㄴ & 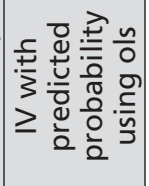 & $\begin{array}{l}0 \\
0 \\
0 \\
0 \\
1 \\
1\end{array}$ & $\begin{array}{l}* \\
* \\
* \\
* \\
\\
0 \\
0 \\
0\end{array}$ & 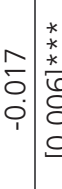 & \begin{tabular}{l|l}
$x$ & \\
$x$ \\
$x$ \\
$\vdots$ \\
$\vdots$ \\
0 \\
0 \\
0 \\
0
\end{tabular} & 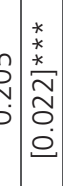 & $\begin{array}{l}0 \\
\stackrel{0}{ } \\
\tilde{0} \\
0\end{array}$ & 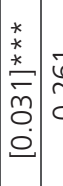 & 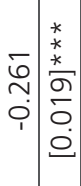 & 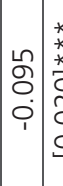 & 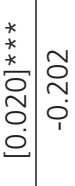 & $\mid \begin{array}{c}* \\
* \\
* \\
* \\
m \\
m \\
m \\
0 \\
\vdots \\
0\end{array}$ & 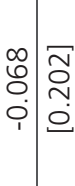 & $\stackrel{\Perp}{\check{2}}$ & & 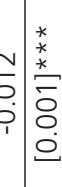 & $\begin{array}{l}\dot{\theta} \\
\dot{0} \\
\dot{0}\end{array}$ & 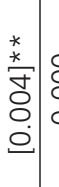 & $\begin{array}{l}8 \\
8 \\
\\
0 \\
\end{array}$ \\
\hline 5 & 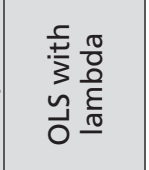 & $\begin{array}{l}0 \\
0 \\
0 \\
0 \\
1\end{array}$ & $\begin{array}{l}* \\
* \\
* \\
+ \\
\\
0 \\
0 \\
0\end{array}$ & 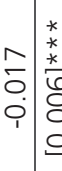 & 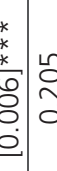 & 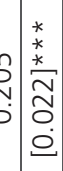 & $\begin{array}{c}\dot{0} \\
\tilde{m} \\
0\end{array}$ & 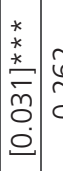 & 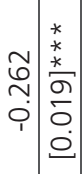 & 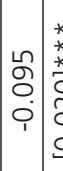 & 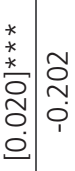 & 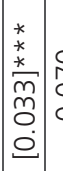 & 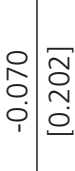 & $\stackrel{\Perp}{\check{0}}$ & & 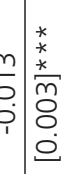 & $\begin{array}{l}9 \\
8 \\
8 \\
0\end{array}$ & \begin{tabular}{l|l}
$\frac{*}{2}$ & \multicolumn{1}{c}{} \\
$\circ$ & \\
$\circ$ & \\
0 &
\end{tabular} & $\begin{array}{l}8 \\
8 \\
\\
\\
\end{array}$ \\
\hline & $\tilde{0}$ & $\mid \begin{array}{l}N \\
\hat{O} \\
0 \\
1 \\
1\end{array}$ & \begin{tabular}{|l|}
$*$ \\
$*$ \\
$*$ \\
$*$ \\
\\
\\
0 \\
0 \\
\end{tabular} & 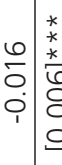 & 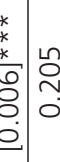 & 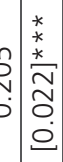 & $\begin{array}{c}\hat{N} \\
m \\
0\end{array}$ & 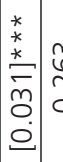 & 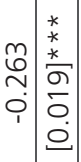 & 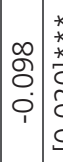 & 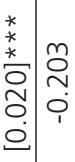 & $\mid \begin{array}{c}* \\
x \\
* \\
* \\
m \\
m \\
0 \\
0 \\
0 \\
0\end{array}$ & 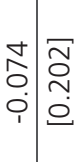 & $\stackrel{\tilde{d}}{\nu^{\prime}}$ & & 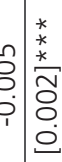 & $\begin{array}{l}10 \\
\vdots \\
0 \\
0\end{array}$ & 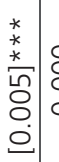 & $\begin{array}{l}8 \\
8 \\
0 \\
\\
1\end{array}$ \\
\hline & 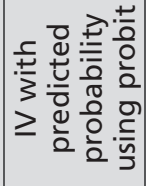 & 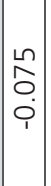 & 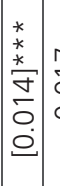 & 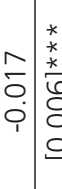 & 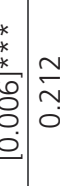 & 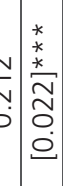 & $\begin{array}{c}\stackrel{0}{2} \\
m \\
0\end{array}$ & 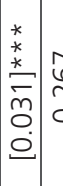 & 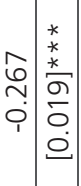 & 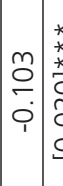 & 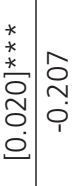 & $\mid \begin{array}{l}* \\
* \\
* \\
* \\
m \\
m \\
m \\
0 \\
0 \\
0\end{array}$ & 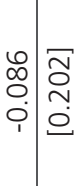 & $\stackrel{\Perp}{\check{\Perp}}$ & & 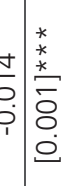 & $\mid \begin{array}{l}\hat{8} \\
8 \\
0\end{array}$ & $\begin{array}{l}\stackrel{*}{*} \\
8 \\
\varnothing \\
0\end{array}$ & $\begin{array}{l}8 \\
8 \\
0 \\
0 \\
1\end{array}$ \\
\hline$\frac{\pi}{\frac{T}{c}}$ & 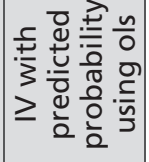 & 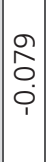 & 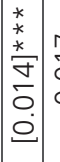 & 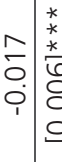 & 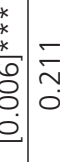 & 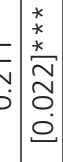 & $\begin{array}{c}\bar{m} \\
m \\
0 \\
0\end{array}$ & 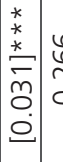 & 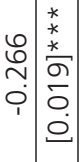 & 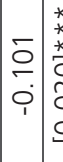 & 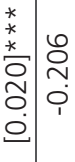 & \begin{tabular}{|l|l}
$*$ & \\
$*$ & \\
$*$ & $\delta$ \\
$m$ & $\delta$ \\
$m$ & \\
0 \\
$\dot{0}$ \\
0
\end{tabular} & 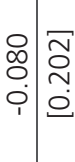 & $\stackrel{\tilde{d}}{\nu}$ & & 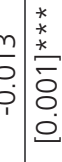 & $\mid \begin{array}{l}\infty \\
0 \\
0 \\
0 \\
0\end{array}$ & 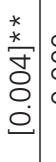 & \\
\hline 5 & $\begin{array}{l}\frac{5}{1} \frac{\pi}{0} \\
3 \frac{0}{1} \\
\frac{1}{0} \frac{0}{0}\end{array}$ & $\begin{array}{c}0 \\
\hat{0} \\
0 \\
1 \\
1\end{array}$ & 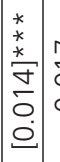 & 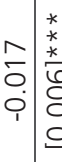 & 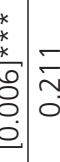 & 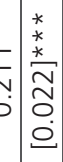 & $\begin{array}{c}\bar{m} \\
m \\
0\end{array}$ & \begin{tabular}{l|l}
$*$ & \\
$*$ & \\
$*$ & $y$ \\
$\bar{\sigma}$ & $y$ \\
$m$ & $c$ \\
0 & $c$ \\
0 &
\end{tabular} & 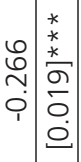 & 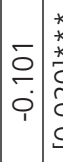 & 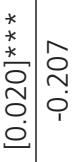 & 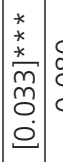 & 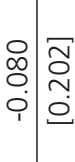 & $\stackrel{\tilde{c}}{\nu}$ & & 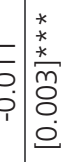 & $\begin{array}{l}0 \\
0 \\
0 \\
0\end{array}$ & \begin{tabular}{l|l}
$*$ & \\
$*$ & \\
& \\
8 \\
$\dot{0}$ \\
0
\end{tabular} & $\begin{array}{l}8 \\
8 \\
0 \\
1\end{array}$ \\
\hline & 0 & $\mid \begin{array}{l}N \\
\hat{0} \\
0 \\
1\end{array}$ & $\mid$\begin{tabular}{l}
$*$ \\
$*$ \\
$*$ \\
$*$ \\
\multirow{\sigma}{*}{} \\
$\vdots$ \\
0 \\
0 \\
0
\end{tabular} & 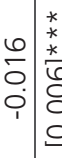 & 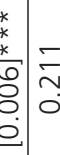 & 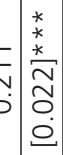 & $\begin{array}{c}\tilde{m} \\
m \\
0 \\
0\end{array}$ & 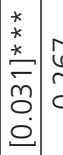 & 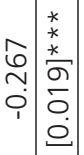 & 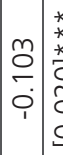 & 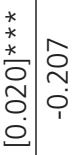 & 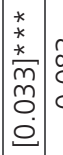 & 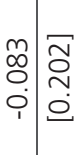 & 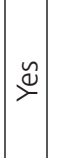 & 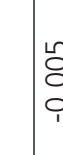 & 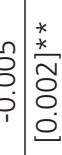 & $\begin{array}{l}0 \\
0 \\
0 \\
0\end{array}$ & 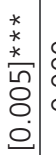 & \\
\hline & & 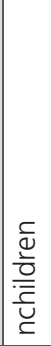 & & 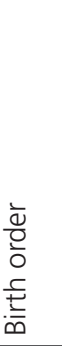 & 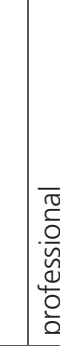 & 产 & 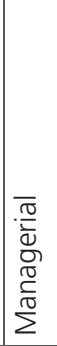 & & 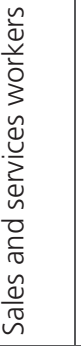 & 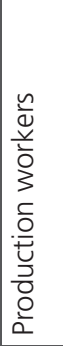 & 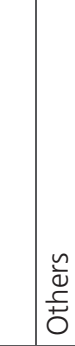 & & 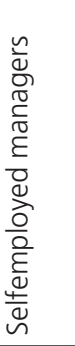 & 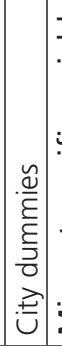 & 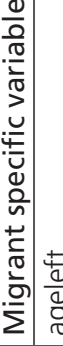 & & 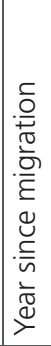 & & 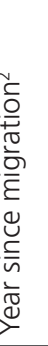 \\
\hline
\end{tabular}




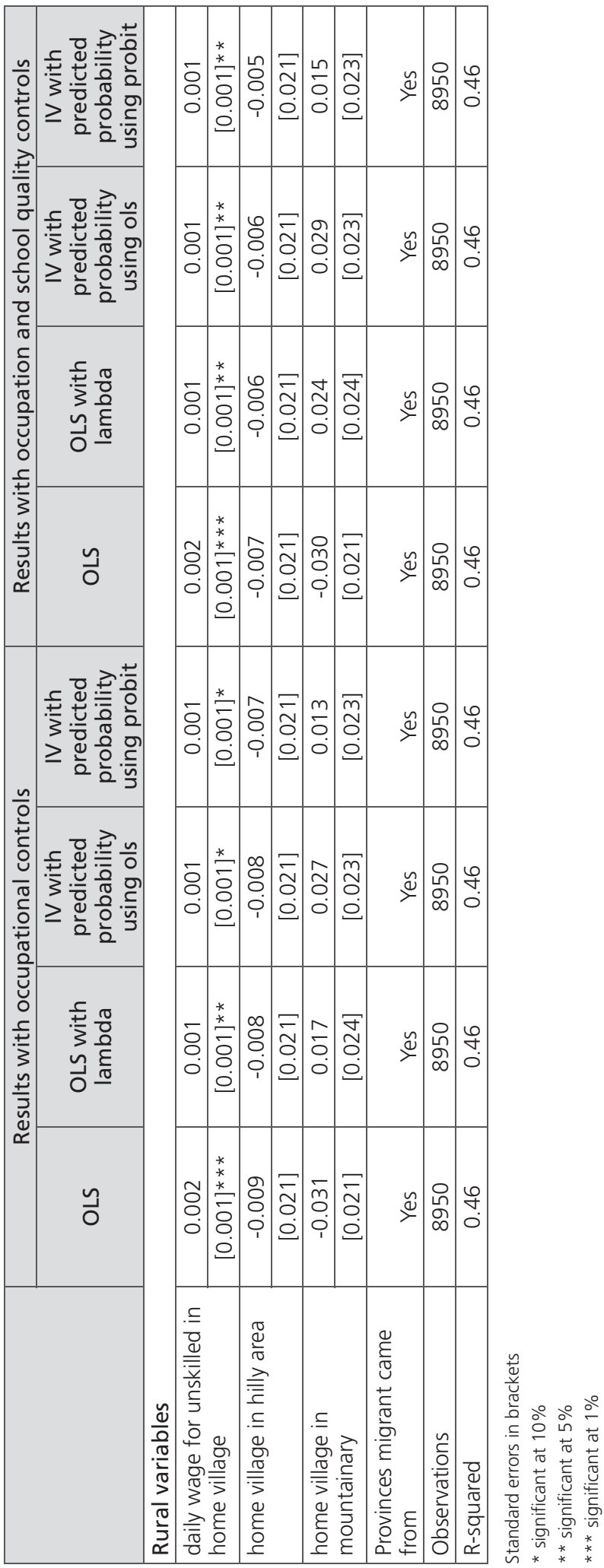




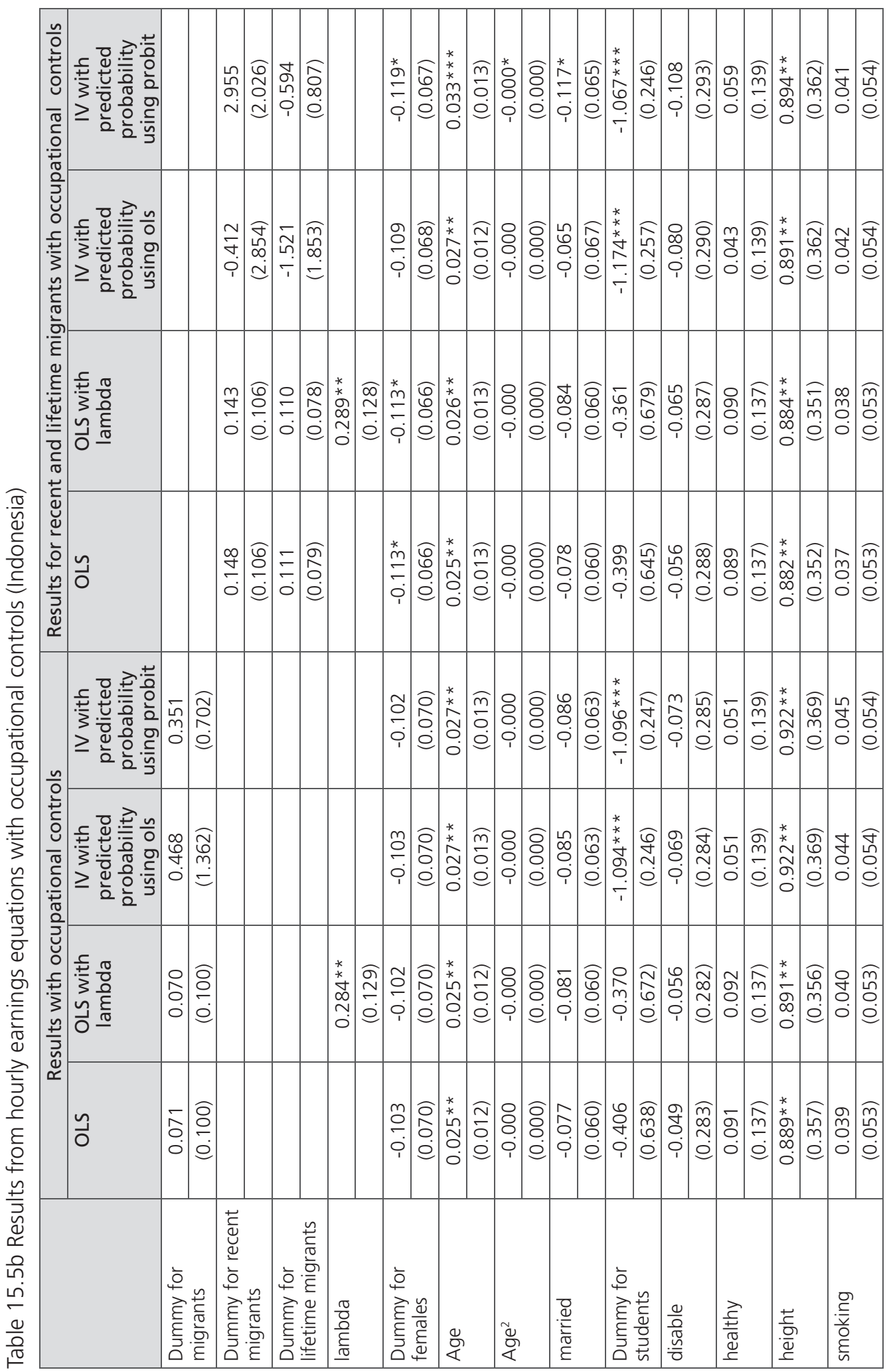




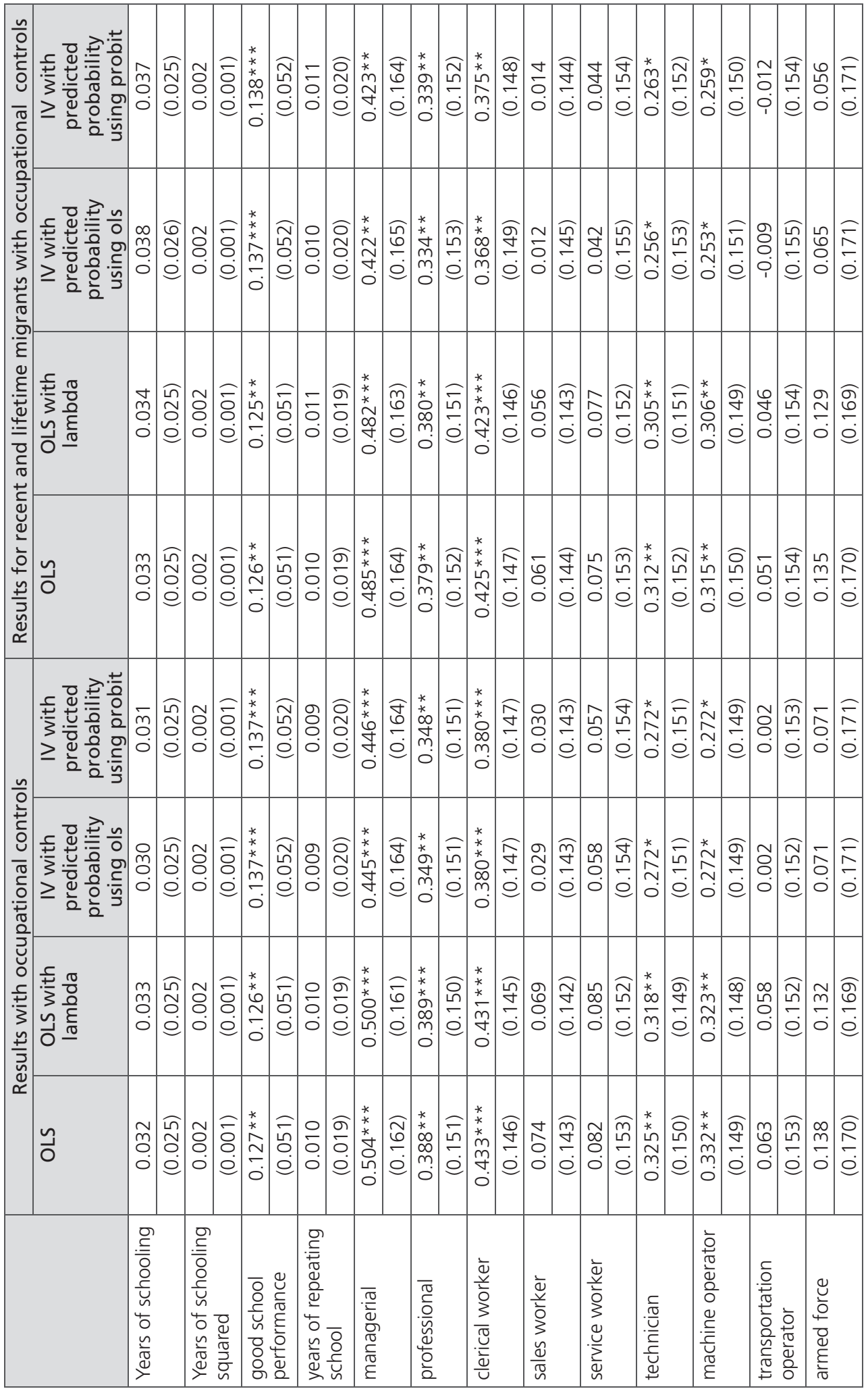




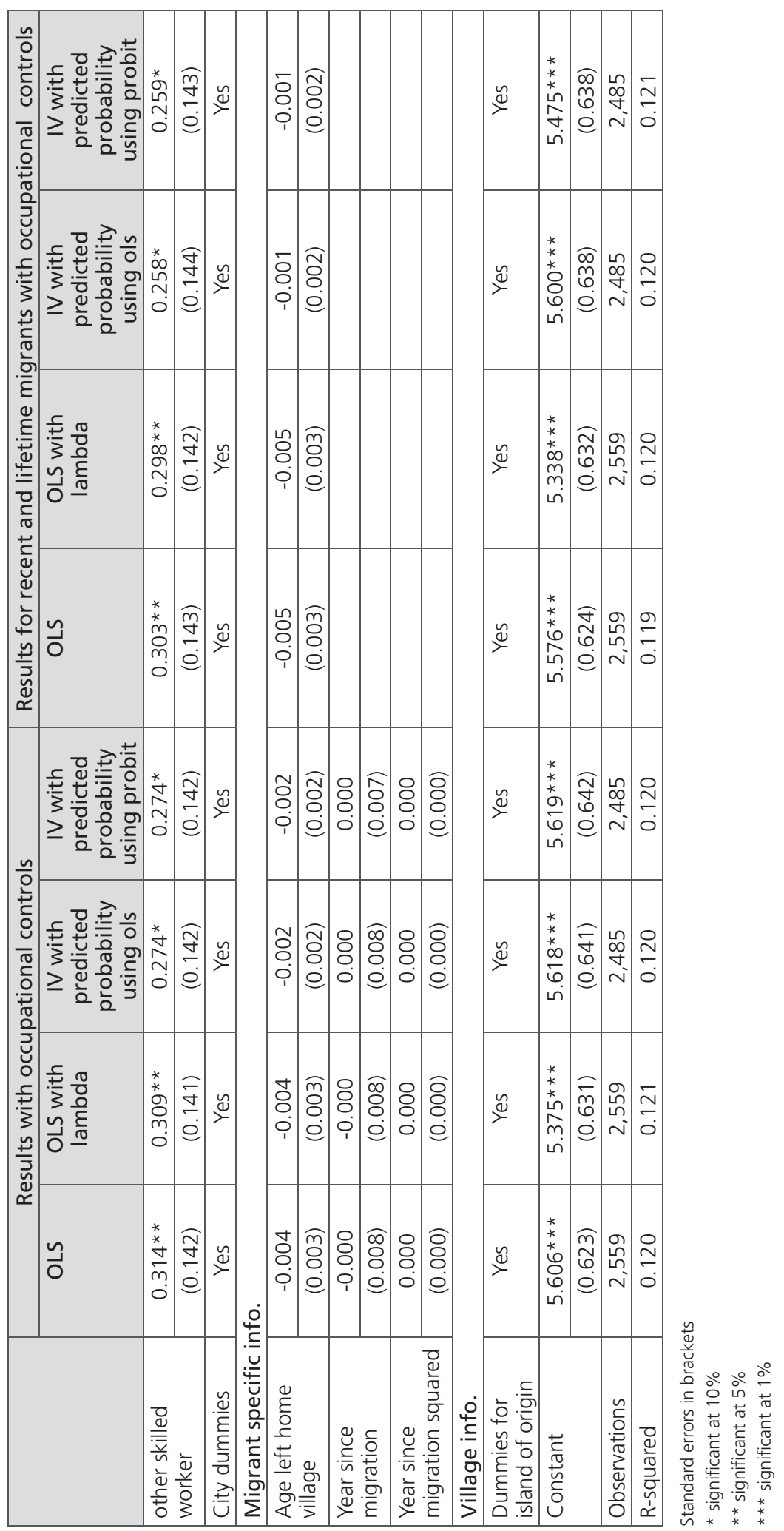




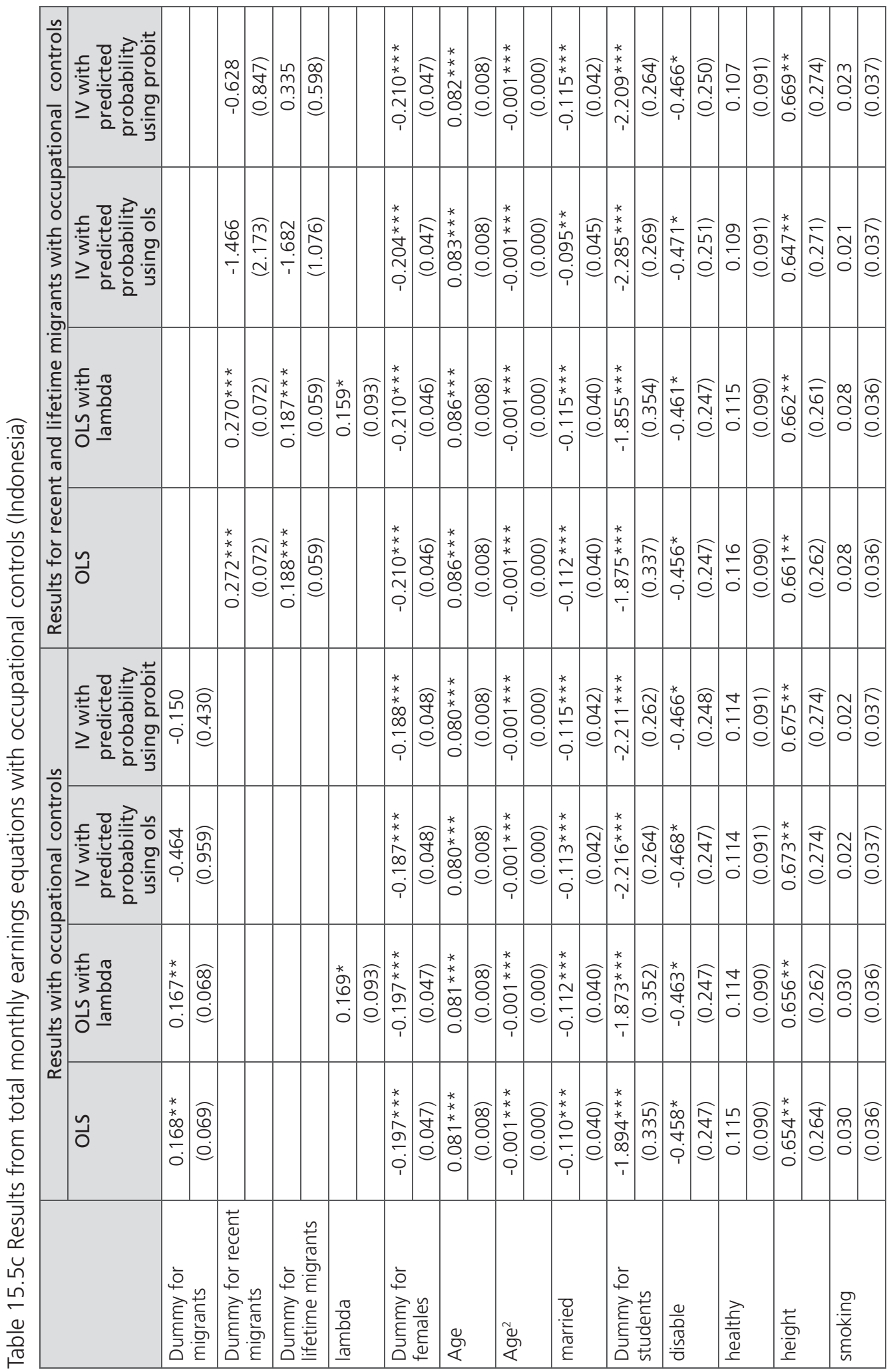




\begin{tabular}{|c|c|c|c|c|c|c|c|c|c|c|c|c|c|c|c|c|c|c|c|c|}
\hline $\begin{array}{l}\frac{ \pm}{ \pm} \\
0 \\
0 \\
\frac{\pi}{0} \\
0\end{array}$ & 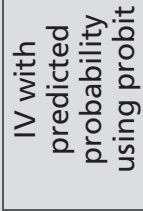 & $\begin{array}{l}* \\
x \\
x \\
* \\
0 \\
0 \\
0 \\
0 \\
0\end{array}$ & 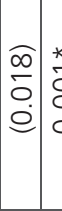 & \begin{tabular}{l|l}
$*$ & $\overline{8}$ \\
$\overline{8}$ & $\overline{8}$ \\
$\dot{0}$ &
\end{tabular} & 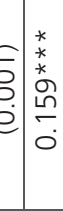 & 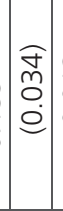 & \begin{tabular}{l|l}
$m$ & $a$ \\
0 & $a$ \\
0 & $c$ \\
0 & $c$
\end{tabular} & 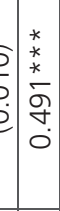 & $\begin{array}{l}\widehat{m} \\
\stackrel{y}{+} \\
\dot{c} \\
\stackrel{c}{c}\end{array}$ & \begin{tabular}{l|l}
0 & $\widehat{n}$ \\
0 & $m$ \\
$\vdots$ & 0 \\
& $\vdots$ \\
\end{tabular} & 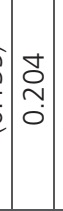 & 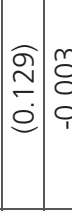 & 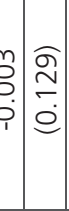 & 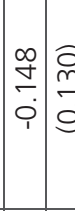 & 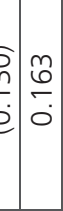 & & \begin{tabular}{l|l}
$\frac{m}{m}$ \\
$\stackrel{m}{m}$ \\
$\vdots$ \\
$\vdots$
\end{tabular} & $\begin{array}{ll}\bar{m} & \\
0 \\
0 \\
0\end{array}$ & & 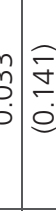 \\
\hline 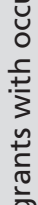 & 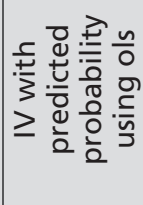 & $\begin{array}{l}* \\
* \\
* \\
* \\
\hat{n} \\
0 \\
0 \\
0\end{array}$ & 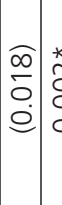 & 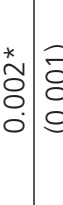 & 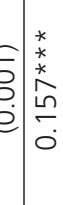 & $\begin{array}{l}\stackrel{f}{\tilde{m}} \\
0 \\
\dot{0}\end{array}$ & $\begin{array}{c}m \\
\overline{0} \\
0 \\
0\end{array}$ & 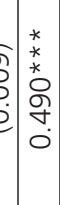 & 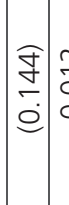 & \begin{tabular}{l|l}
$N$ & $\widehat{0}$ \\
0 & $m$ \\
$\dot{0}$ & $\vdots$ \\
1 &
\end{tabular} & $\mid \begin{array}{l}\checkmark \\
\stackrel{2}{*} \\
0\end{array}$ & 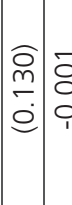 & 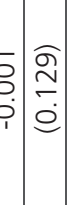 & 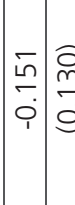 & 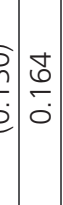 & & 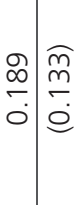 & $\begin{array}{c}m \\
m \\
0 \\
0 \\
0\end{array}$ & & 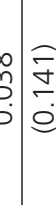 \\
\hline 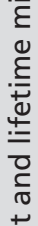 & 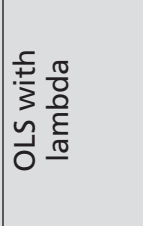 & $\begin{array}{l}* \\
* \\
* \\
* \\
N \\
0 \\
0 \\
0\end{array}$ & 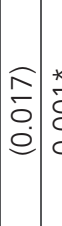 & 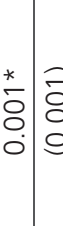 & 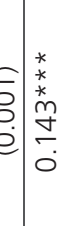 & $\begin{array}{c}\widehat{f} \\
m \\
0 \\
\dot{e}\end{array}$ & 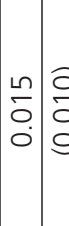 & 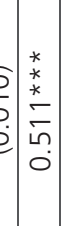 & 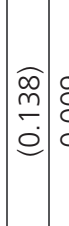 & 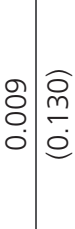 & {$\left[\begin{array}{c}* \\
0 \\
\\
N \\
0\end{array}\right.$} & $\begin{array}{l}\stackrel{\overbrace{}}{\stackrel{a}{a}} \\
\stackrel{a}{c} \\
\check{c}\end{array}$ & 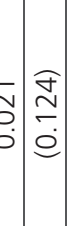 & & 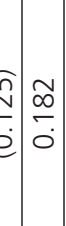 & & 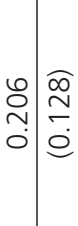 & 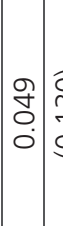 & & 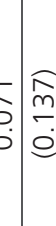 \\
\hline 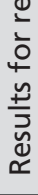 & ○े & \begin{tabular}{l}
$*$ \\
$*$ \\
$*$ \\
$*$ \\
\multirow{2}{0}{} \\
0 \\
0
\end{tabular} & 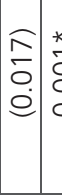 & 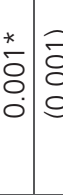 & 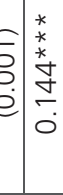 & $\begin{array}{c}\text { f } \\
m \\
0 \\
\dot{c}\end{array}$ & 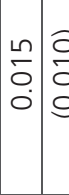 & 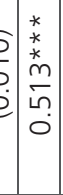 & 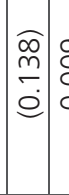 & 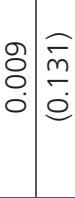 & $\mid \begin{array}{c}* \\
\tilde{J} \\
\sim \\
0 \\
0\end{array}$ & 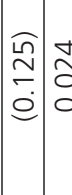 & 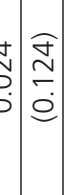 & 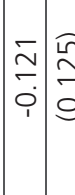 & 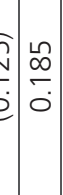 & & 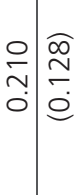 & 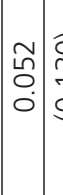 & & 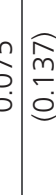 \\
\hline & 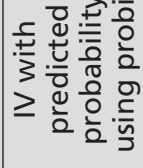 & 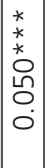 & 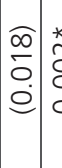 & 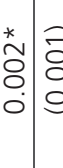 & 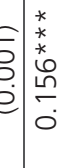 & $\begin{array}{l}\text { f } \\
\tilde{m} \\
\dot{0} \\
\end{array}$ & 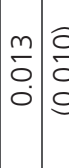 & 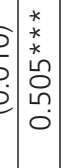 & 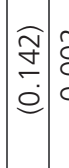 & 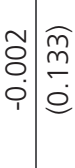 & {$\left[\begin{array}{c}0 \\
\\
0 \\
0\end{array}\right.$} & 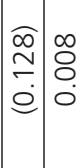 & 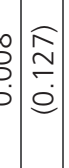 & 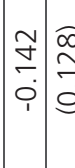 & 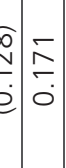 & & $\begin{array}{ccc}\bar{\delta} & \bar{m} \\
\underset{n}{c} \\
\dot{e}\end{array}$ & \begin{tabular}{c|c}
$\hat{n}$ & $\hat{n}$ \\
0 \\
0 \\
0
\end{tabular} & & 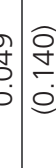 \\
\hline $\begin{array}{l}\frac{5}{0} \\
\frac{0}{0} \\
\frac{0}{+}\end{array}$ & 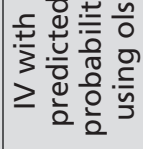 & 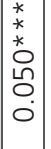 & 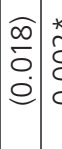 & 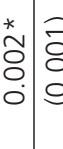 & 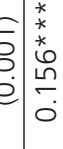 & $\begin{array}{l}\widehat{f} \\
m \\
0 \\
\dot{0}\end{array}$ & $\begin{array}{lll}m & a \\
0 & a \\
0 & c \\
0 & c & \end{array}$ & 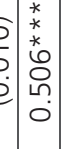 & 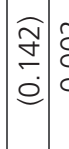 & 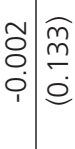 & $\hat{\imath}$ & 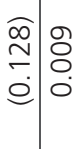 & 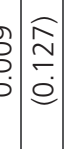 & $\begin{array}{ll}\bar{f} & \infty \\
\dot{q} \\
\dot{q}\end{array}$ & 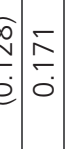 & & 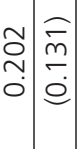 & 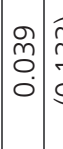 & & مُ \\
\hline 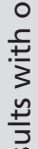 & 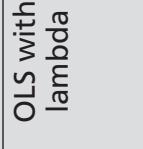 & \begin{tabular}{|l|}
$*$ \\
$*$ \\
$*$ \\
$*$ \\
$N$ \\
0 \\
0 \\
0
\end{tabular} & $\begin{array}{l}\infty \\
\overline{0} \\
\dot{e}\end{array}$ & $\begin{array}{l}\overline{8} \\
\overline{8} \\
\dot{0}\end{array}$ & 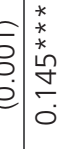 & $\begin{array}{c}\widehat{f} \\
\grave{m} \\
0 \\
\varrho\end{array}$ & $\begin{array}{ll}10 & 0 \\
0 & 0 \\
0 & 0 \\
0 & 0\end{array}$ & 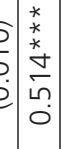 & $\mid \begin{array}{l}\widehat{\infty} \\
\stackrel{m}{c} \\
\stackrel{c}{e}\end{array}$ & 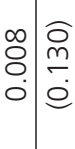 & $\begin{array}{c}* \\
\\
N \\
0\end{array}$ & 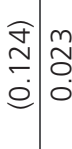 & 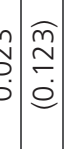 & 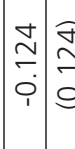 & 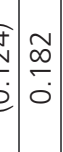 & & 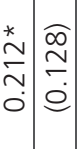 & 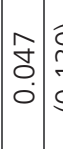 & & 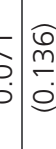 \\
\hline & 0 & 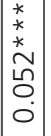 & $\begin{array}{c}0 \\
0 \\
0 \\
0\end{array}$ & $\begin{array}{l}\bar{\delta} \\
\bar{\delta} \\
\dot{0}\end{array}$ & 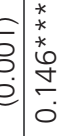 & $\begin{array}{c}\widehat{f} \\
\tilde{m} \\
0 \\
\dot{c}\end{array}$ & 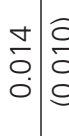 & 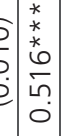 & 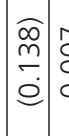 & 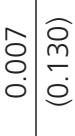 & $\mid \begin{array}{l}* \\
\infty \\
m \\
N \\
0\end{array}$ & 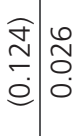 & 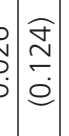 & 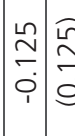 & 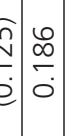 & 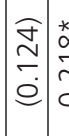 & 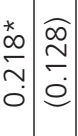 & \begin{tabular}{ll}
0 & 1 \\
\hdashline & $\vdots$ \\
0 & $\vdots$ \\
0
\end{tabular} & & 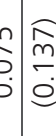 \\
\hline & & 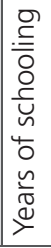 & & 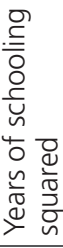 & 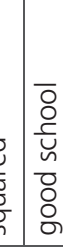 & 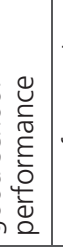 & 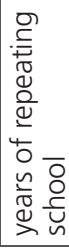 & 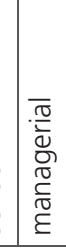 & & 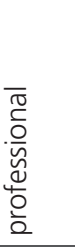 & 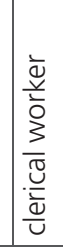 & $\begin{array}{l}\bar{a} \\
\frac{y}{0} \\
\frac{3}{0} \\
\frac{u}{v}\end{array}$ & & 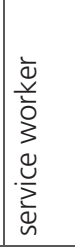 & 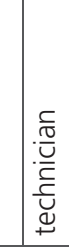 & & 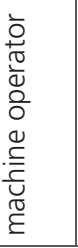 & 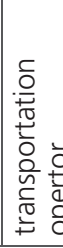 & 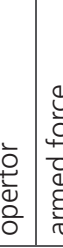 & है \\
\hline
\end{tabular}




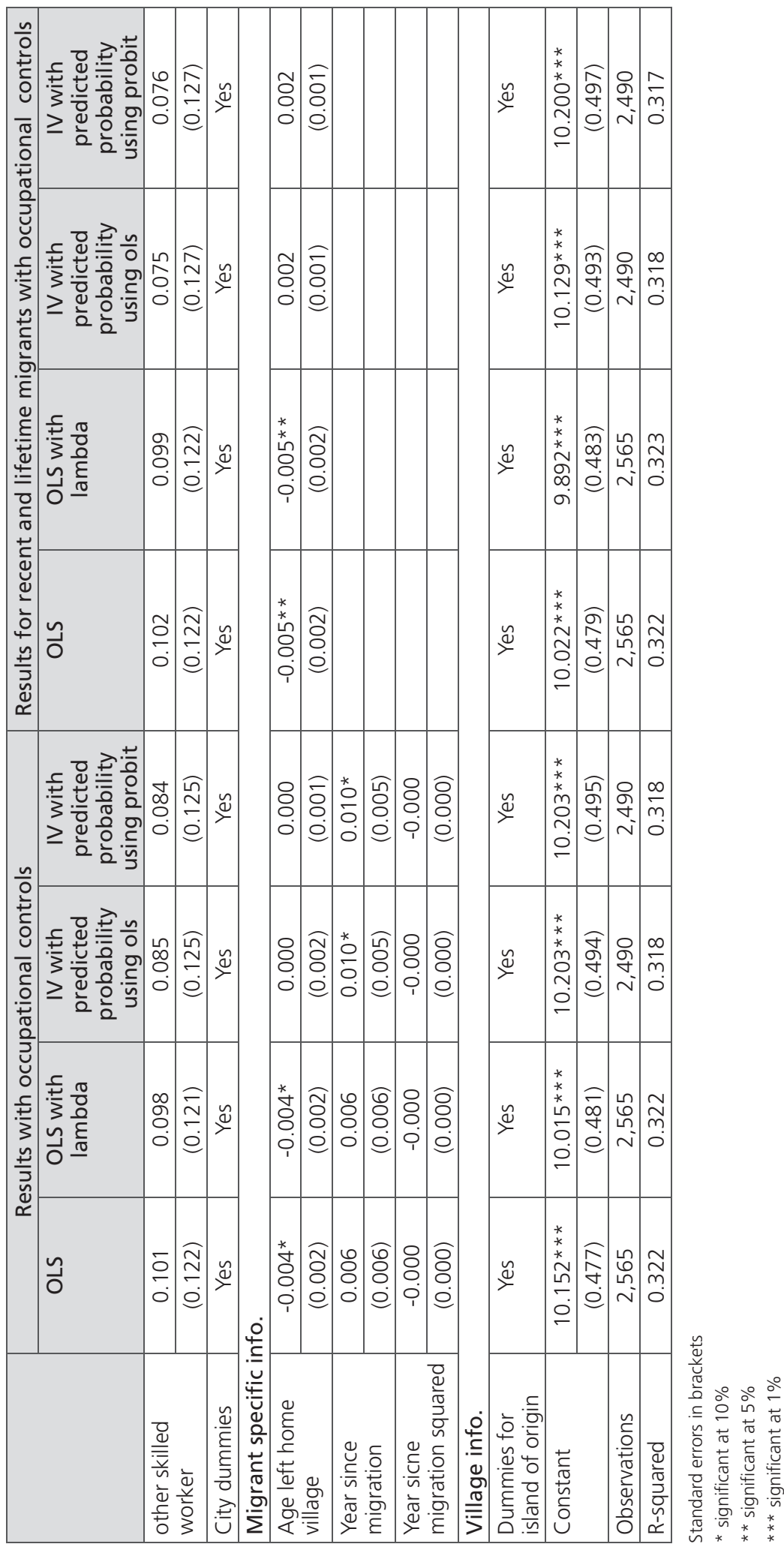




\section{Conclusions}

This chapter examined wage differentials between migrants and urban individuals in China and Indonesia, with particular interest in the role of occupational restrictions. Migrants in China were found to receive nearly 50 per cent lower hourly wages than their urban counterparts, of which close to 20 per cent could be explained by differential access to high-wage occupations. Almost the opposite held in Indonesia, which has a laissez-faire policy towards migrants within cities. In Indonesia, recent migrants earn about 6 per cent more than those born in the city, but the majority of this small difference is captured by the fact that they are over-represented in high-paying occupations, which one can interpret either as a sign of compensating differentials (migrants willingly trade-off wages for less pleasant types of jobs) or as a greater willingness to work hard (which is also witnessed by their higher hours of work). In both countries, migrants tend to be relatively young and better educated. Also, in both countries there is evidence of the positive selectivity of migrants in that some 12-13 per cent of the earnings of migrants in both countries come from positive characteristics (higher ability) that migrants possess but that non-migrants in the countryside do not possess.

The overall message for policy makers is that the internal tensions in the Chinese system are far greater than they are in Indonesia. Indeed, there is no evidence of systematic discrimination or even of a relative backwardness of migrants in Indonesian cities, in which very similar wages and occupational distributions are observed for migrants and non-migrants. From a labour-market point of view, there are hence no clear migrant-specific issues to address in the Indonesian case. In principle, the Indonesian cities can try to mimic the Chinese example of restricting access to the more desirable jobs to current urban residents, but this would need a level of organisation and political cohesion that seems unlikely to occur soon.

The opposite is true in China. A very large degree of outright discrimination is observed that does not seem sustainable in the long run given the ethos of the Chinese state bureaucracy as being the custodian of the welfare of all Chinese. The implicit current taxation of the migrants by urban incumbents, via entry restrictions for migrants into higher wage occupations such as the civil service and managerial positions, might benefit urban incumbents and might even keep export prices down, but this comes at the expense of the earnings levels of migrants and thereby also of those who depend on those migrants in the countryside. As China's urbanisation process is an unstoppable trend in line with the development experience in other countries, it seems unworkable to have an explicit two-class residency system inside the cities in the long run. The policy question then becomes how to make the playing field more level over time. One way is for the urban insiders to change the rules within the cities as to how to become an official resident.

By changing the access to urban hukou, urban insiders may explicitly sell off the right to be urban - something that already occurs at the moment because the most successful migrants manage to buy themselves into the club of urban hukou holders (and via marriage) but at a price that is simply too high for the majority of migrants. The laissez-faire solution is to wait for that price to drop as urban incumbents cash in on their current position of power in deciding who can be urban, by selling off these rights in larger chunks (for short--term 
gain but with longer-term equality as the outcome). Competition between cities, which will intensify when the migrant streams run dry, will speed up the price reductions in urban residency. Given that there are still some 800 million people living in the countryside, this market solution might take a few decades still. The centralised policy solution would be to try to legislate towards more equal treatment, in the form of centralised education and health programs (such that individual cities do not control access to urban education or health) and centralised inspectorates to enforce equal opportunity legislation. Either way, the centralised solution essentially entails a power struggle between the centre and the cities, and will continue to challenge policy makers across China for some time to come.

\section{Bibliography}

AFX NEWS 2005, 'China's Hu says political stability helps maintain high economic growth', AFX NEWS, 15 October 2005, <http://www.forbes.com/markets/feeds/afx/2005/10/15/ afx2278991.html>

Anderson, J. 2005, How to Think About China, UBS Securities Asia Limited, Hong Kong.

Banerjee, B. 1984, 'Information flow, expectations and job search', Journal of Development Economics, vol. 15, pp. 239-57.

Baum, R. 1992, 'Political stability in post-Deng China: problems and prospects', Asian Survey, vol. 32, no. 6, pp. 491-505.

Becker, G. 1971, The Economics of Discrimination, [Second edition], University of Chicago Press, Chicago.

Bell, M. and Muhidin, S. 2009, Cross-national comparison of internal migration, UNDP Research Paper 2009/30, United Nations Development Programme, New York.

Borjas, G. J. 2003, 'The labor demand curve is downward sloping: re-examining the impact of immigration on the labor market', Quarterly Journal of Economics, vol. 118, no. 4, pp. 1335-74.

Brunnermeier, M. and Parker, J. 2004, Optimal expectations, NBER Working Paper 10707, National Bureau of Economic Research, Cambridge, Mass., <www.nber.org/papers/ w10707>

Cannon, W. B. 1914, 'Recent studies of bodily effects of fear, rage, and pain', Journal of Philosophy, Psychology and Scientific Methods, vol. 11, no. 6, pp. 162-5.

Card, D. 2009, Immigration and inequality, NBER Working Paper No. 14683, National Bureau of Economic Research, Cambridge, Mass.

Card, D. E., Dustmann, C. and Preston, I. 2009, Immigration, wages, and compositional amenities, NBER Working Paper No. w15521, (November 2009), National Bureau of Economic Research, Cambridge, Mass.

Clark, A., Frijters, P. and Shields, M. A. 2008, 'A survey of the income happiness gradient', Journal of Economic Literature, vol. 46, no. 1 (March), pp. 95-144. 
Das, M. and van Soest, A. 1999, 'A panel data model for subjective information on household income growth', Journal of Economic Behavior and Organization, vol. 40, no. 4, pp. 409-26.

Du, Y., Gregory, R.G., and Meng, X. 2006, 'Impact of the guest worker system on poverty and wellbeing of migrant workers in urban China' in Ross Gaunaut and Ligang Song (eds), The Turning Point in China's Economic Development, Asia Pacific Press, Canberra.

Easterlin, R. A. 2001, 'Income and happiness: towards a unified theory', Economic Journal, vol. 111, pp. 465-84.

Ferrer-i-Carbonel, A. and Frijters, P. 2004, 'The effect of methodology on the determinants of happiness', Economic Journal, vol. 114, pp. 641-59.

Frijters, P., Shields, M. A. and Haisken-DeNew, J. P. 2006, ‘How rational were expectations in East Germany after the falling of the wall?', Canadian Journal of Economics, IZA working paper.

Giessmann, H. J. 2007, ChIndian and ASEAN: about national interests, regional legitimacies, and global challenges, FES Briefing Paper 7, Friedrich Ebert Stiftung, Berlin.

Hagerty, M. 2003, 'Was life better in the "good old days"? Intertemporal judgments of life satisfaction', Journal of Happiness Studies, vol. 4, pp. 115-39.

Hamermesh, D. S. 2004, Subjective outcomes in economics, NBER Working Paper No. W10361, National Bureau of Economic Research, Cambridge, Mass.

Harwood, K., MacLean, N. and Durkin, K. 2007, 'First-time mothers' expectations of parenthood: what happens when optimistic expectations are not matched by later experiences?', Developmental Psychology, vol. 43, no. 1, pp. 1-12.

Holbig, H. 2006, 'Ideological reform and political legitimacy in China: challenges in the post-Jiang era', German Institute of Global and Area Studies, no. 18.

Lu, X. 2003, 'Corruption and regime legitimacy in China', in F. Godement (ed.), China's New Politics, La Documentation Francais, Paris.

Mankiw, N. G., Reis, R. and Wolfers, J. 2003, Disagreement about inflation expectations, NBER Working Papers 9796, National Bureau of Economic Research, Cambridge, Mass..

Meng, X. 2000, ‘Regional wage gap, information flow, and rural-urban migration', in Y. Zhao and L. West (eds), Rural Labor Flows in China, University of California Press, Berkeley, pp. $251-77$.

Meng, X. and Manning, C. 2010, 'The Great Migration in China and Indonesia-trend and institutions' in X. Meng and C. Manning, with S. Li, and T.Effendi (eds). The Great Migration: Rural-Urban Migration in China and Indonesia, Edward Elgar Publishing Ltd.

Meng, X. and Zhang, J. 2001, 'The two-tier labour market in urban China: occupational segregation and wage differentials between urban residents and rural migrants in Shanghai', Journal of Comparative Economics, vol. 29, pp. 485-504.

Ottaviano, G. and Peri, G. 2006, Rethinking the effects of immigration on wages, NBER Working Paper No. 12497, National Bureau of Economic Research, Cambridge, Mass. 
Perry, E. and Selden, M. 2000, Chinese Society: Change, conflict and resistance, Routledge, London.

Rabin, M. 1998, 'Psychology and economics', Journal of Economic Literature, vol. 36, pp. 11-46.

Senik, C. 2004, 'When information dominates comparison: a panel data analysis using Russian subjective data', Journal of Public Economics, vol. 88, pp. 2099-123.

Song, L. and Appleton, S. 2008, Life satisfaction in urban China: components and determinants, IZA Discussion Papers 3443, Institute for the Study of Labor, Bonn.

Souleles, N. 2004, 'Expectations, heterogeneous forecast errors, and consumption: micro evidence from the Michigan Consumer Sentiment Surveys', Journal of Money, Credit, and Banking, vol. 36, pp. 39-72.

Stutzer, A. 2004, 'The role of income aspirations in individual happiness', Journal of Economic Behavior and Organization, vol. 54, no. 1, pp. 89-109.

Veenhoven, R. 2004, 'Average happiness in 90 nations 1990-2000', World Database of Happiness, RankReport 2004/lc, <worlddatabaseofhappiness.eur.nl>

West, L. and Zhao, Y. (eds). 2000, Rural Labor Flows in China, University of California Press, Berkeley.

World Bank 2009, Development Report 2009, The World Bank, Washington, DC.

Younis, M., Lin, X. X., Sharahili, Y. and Selvarathinam, S. 2008, 'Political stability and economic growth in Asia', American Journal of Applied Sciences, vol. 5, no. 3, pp. 203-8.

Zeng, S. 2003, 'Leadership change, legitimacy, and party transition in China', Journal of Chinese Political Science, vol. 8, nos 1-2 (September), pp. 47-63.

\section{Endnotes}

1. The few consequences of residential rights are that one may own a vehicle with a local number plate, and one may own a property in the area of the residency right. Given the large market for rented accommodation, these benefits are small compared with those in China. A restriction of access to residential cards in a few large cities, such as Jakarta, is that to obtain a residential card in these cities one needs to show proof of being employed in a formal private or government institution located in those cities or of being a student in those cities.

2. This identifying assumption also means that in the selection estimation, we need (after weighting) representative proportions of migrants and non-migrants in each region.

3. The nine provinces where the rural survey was conducted are: Guangdong, Jiangsu, Hangzhou, Ningbo, Hefei, Bangbu, Zhengzhou, Luoyang, Chongqing, Chengdu and Wuhan.

4. Available on request from the authors. 